
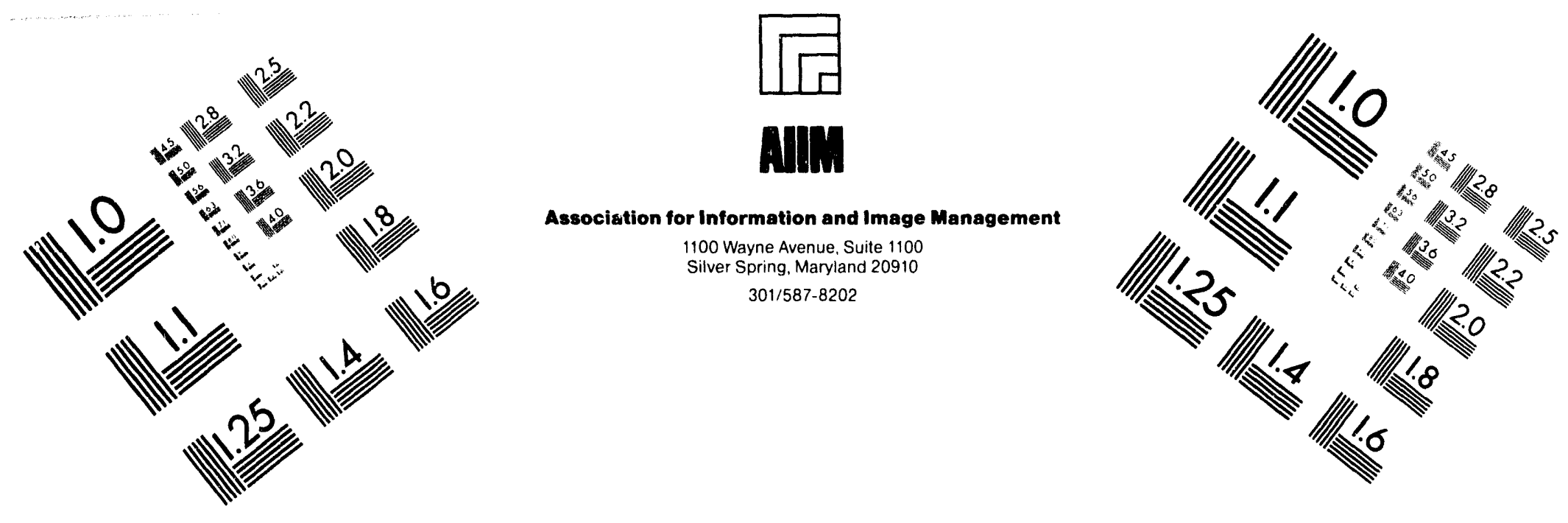

\title{
Centimeter
}

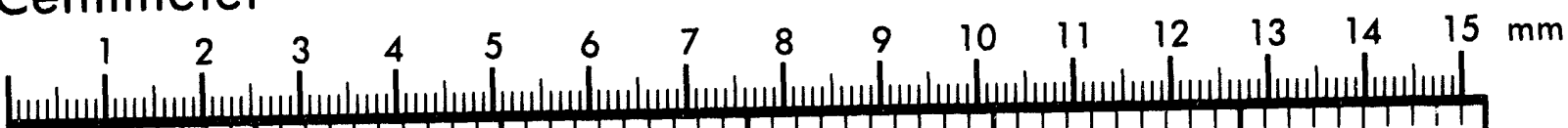

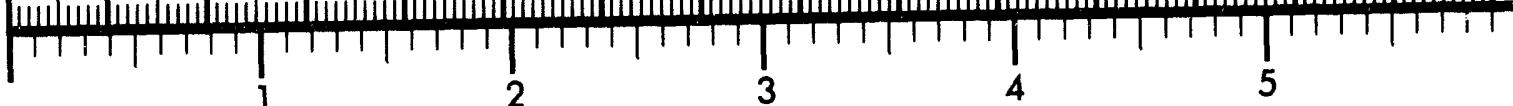
Inches
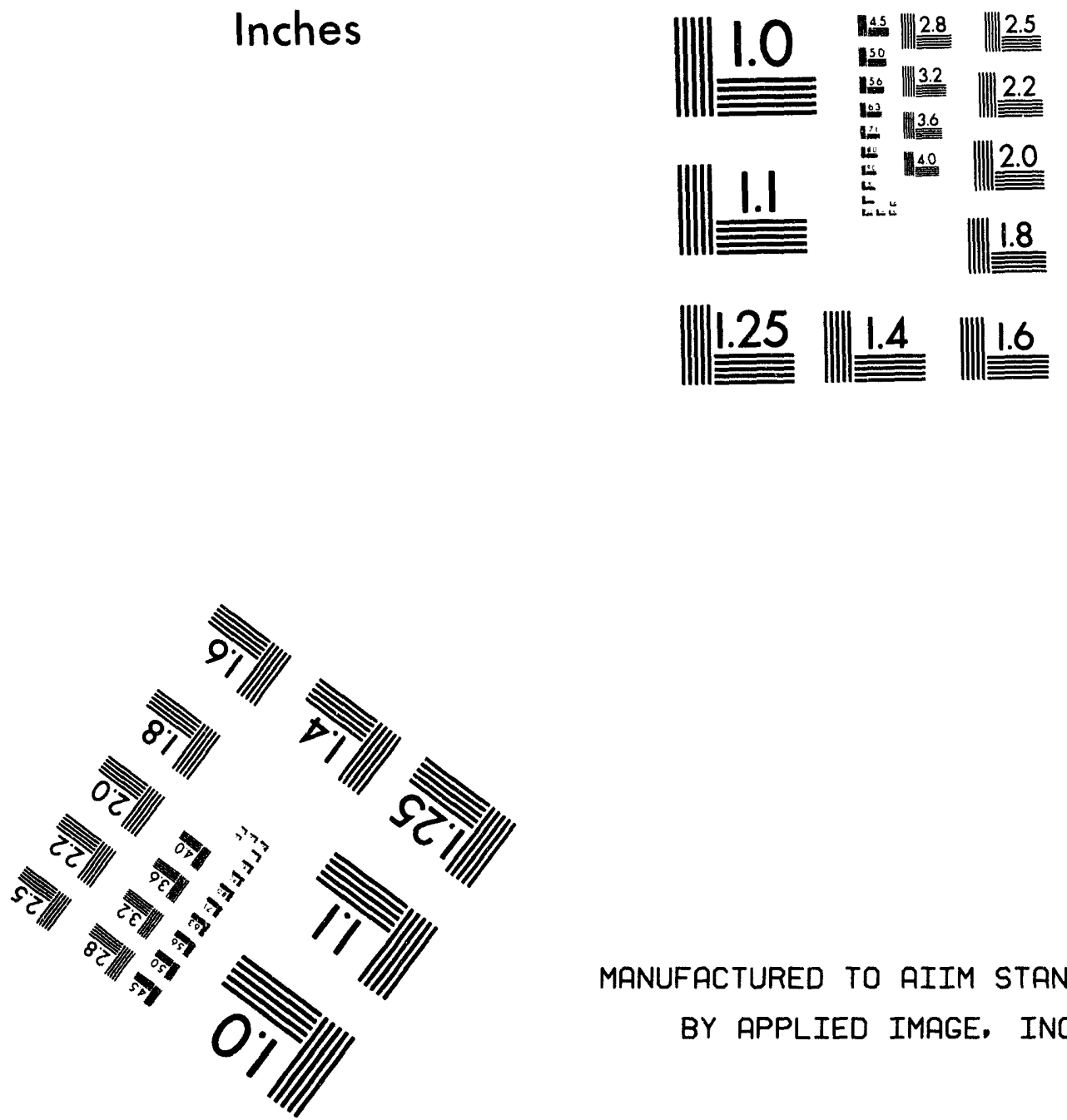

MANUFACTURED TO AIIM STANDARDS

BY APPLIED IMAGE, INC.

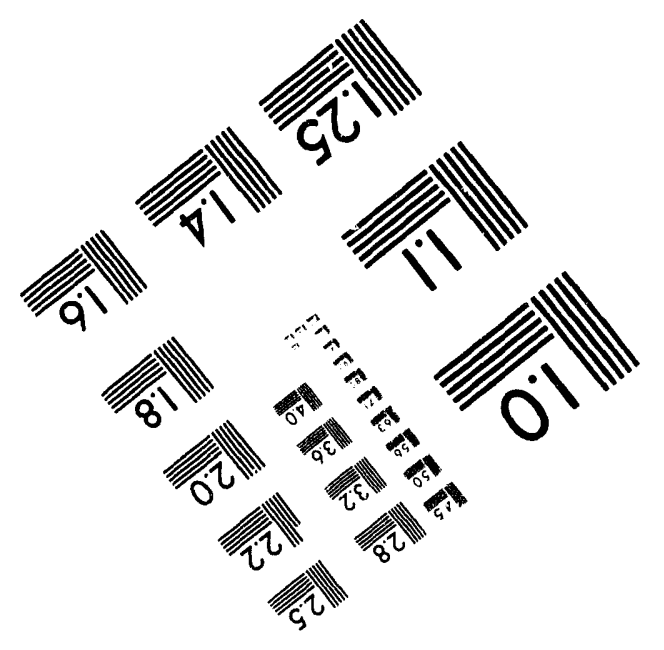



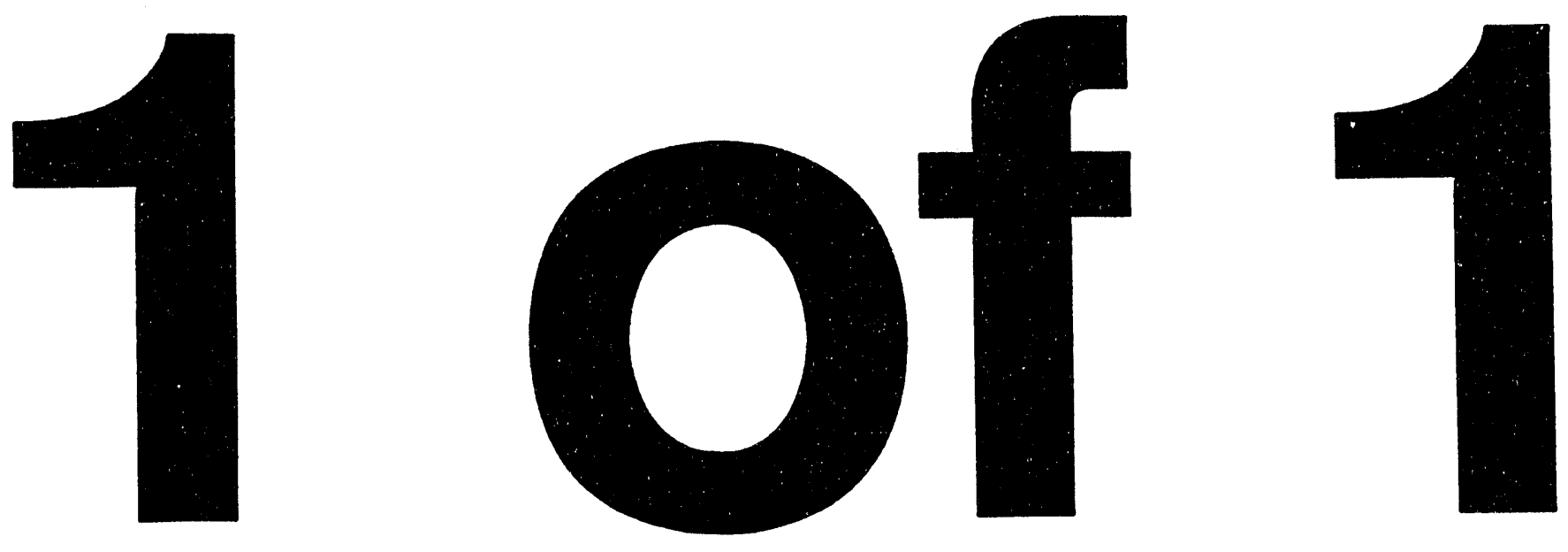


\section{STUDIES OF TRANSVERSE COHERENT BUNCH INSTABILITIES FOR THE ADVANCED LIGHT SOURCE (ALS) *}

M. Meddahi and J. Bengtsson

Center for Beam Physics Accelerator and Fusion Research Division

Lawrence Berkeley Laboratory

University of California

Berkeley, CA 94720

May 31, 1994

*This work was supported by the Director, Office of Energy Research, Office of High Energy and Nuclear Physics, High Energy Physics Division, of the U.S. Department of Energy under Contract No. DE-AC03-76SF00098. 


\begin{abstract}
We have studied the transverse coherent bunch instabilities for the Advanced Light Source (ALS). We have in particular applied a Hamiltonian formalism to obtain the linearized averaged equations of motion (i.e. the one turn map) for the resistive wall effect to obtain the corresponding localized kick when the beta function is varying along the lattice. We have also included a 2 dimensional model for the transverse higher order cavity modes. In addition, we have used power series maps to represent the lattice which enabled us to include non-linear effects. These models have been implemented in a computer code and numerical simulations have been carried out for ALS. The model was successfully verified against analytical calculations in cases where they overlap. The non-linear effects from the lattice proved to be important, since they led to a qualitative change of the dynamics for the stored beam. We also studied the injection process in some detail and found that the non-linear effects also fundamentally change the injection dynamics.
\end{abstract}




\section{Contents}

1 INTRODUCTION 1

2 THE EQUATIONS OF MOTION 2

2.1 The Linear Transverse Equation of Motion . . . . . . . . . . . 2

2.2 Wake Fields and Wake Potentials ............ 4

2.3 Transverse Equation of Motion with Wake Fields . . . . . . 8

2.4 Equation of Motion for a Bunch . . . . . . . . . . . . . . 10

2.5 Transverse Impedance . . . . . . . . . . . . . . . . 12

2.6 Dispersion Relations . . . . . . . . . . . . . . 14

3 THE FACILITIES $\quad 16$

3.1 The Advanced Light Source . . . . . . . . . . . . . . 16

3.2 Description of the Injection Process . . . . . . . . . . . 16

4 THE TRACKING CODE 20

5 THE CAVITY MODEL 22

5.1 The Radio Frequency System . . . . . . . . . . . . . 22

5.2 Cavity Wakefield and Computer Implementation . . . . . . . 22

5.3 Dimensional Analysis . . . . . . . . . . . . . . 26

5.4 Growth Rate . . . . . . . . . . . . . . . . 27

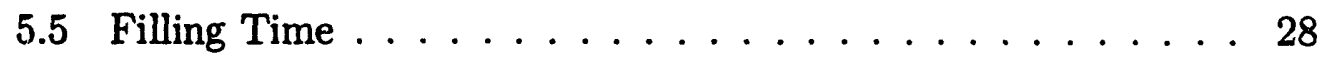

5.6 Scaling of the Quality Factor . . . . . . . . . . . . 28

6 THE RESISTIVE WALL PARAMETERS 29

6.1 Definition ....................... 29

6.2 Resistive Wall Parameter for the ALS and Computer Imple-

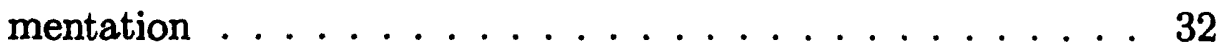

7 PRODUCTION AND ANALYSIS 33

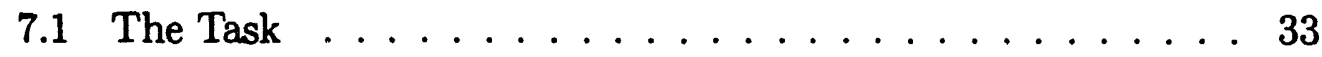

7.2 Linear Map . . . . . . . . . . . . . . . . 35

7.3 Non-Linear Map . . . . . . . . . . . . . . . 38

8 MORE ON THE INJECTION PROCESS 41 
9 OTHER APPLICATIONS 43

10 SUMMARY AND CONCLUSIONS 44 


\section{INTRODUCTION}

Studies of wake fields have a long history in the area of accelerators see e.g. $[1,2,3,4,5]$. The underlying reason is that a beam of charged particles will interact electromagnetically with its environment and in particular, potentially excite wake fields in any electrically or magnetically polarizable or conductive material in the neighborhood of the beam. Since the design and construction of accelerators is essentially a confinement problem, the question then arises how this will affect the performance of the system, e.g. stability, bunch dimensions, heating etc. Since one is in this case dealing with a system of interacting particles it is ultimately on a microscopic level described by quantum field theory, more specifically quantum electrodynamics. However, such an approach would not take us very far. For relevant studies a macroscopic point of view is highly adequate, leading to Maxwell's equations.

The wake fields excited by a charge are in general functions of the spacetime coordinates of both the exciting charge and the point of evaluation. This general but complicated description can be simplified by introducing wake potentials, obtained by integrating the Lorentz force at a certain distance behind the exciting charge. Note however, that this leads for a ring in general to a perturbative treatment since the motion of the exciting charge has to be known. Furthermore, if one only considers the effect of a given wake field on other particles, neglecting the individual particles effect on the wake field, i.e. external field approximation, one may use a Hamiltonian formulation. This also allows for a straightforward introduction of lumped circuit concepts such as impedance originating in electrical engineering from circuit analysis of linear, passive networks with periodic signals (e.g. electronic circuit theory and microwave technology) by generalizing to linear, passive electromagnetic systems with aperiodic signals replacing Fourier series with Fourier transforms.

This paper consist of two parts. The first part outlines the theory from a one turn map point of view using a Hamiltonian formalism. This allows for a more straightforward and transparent formalism in the case of distributed wake fields than direct averaging of the equations of motion as customary. In particular, it allows us to develop a 6-dimensional computer model that includes non-linear effects from the lattice. The second part describes a 2dimensional model for the higher order modes (HOMs) of a cavity as well as the resistive wall effect and how these models have been implemented in a 
tracking code used to study the transverse dynamics for the Advanced Light Source (ALS). Related system parameters are presented and the injection process is described in some detail. Results from the tracking code for various cases are presented and compared against analytical formulas when possible. Qualititave modifications of the dynamics due to non-linear effects from the lattice are also presented. A third part in progress will address detailed tracking studies of the dynamics including related feedback systems.

\section{THE EQUATIONS OF MOTION}

\subsection{The Linear Transverse Equation of Motion}

In the following we will replace the effect of a general wakefield distributed along a periodic lattice by a localized kick. This is done by first determining the action-angle variables for the unperturbed motion followed by averaging the corresponding perturbed Hamiltonian with respect to $s$. This is equivalent to determining the corresponding Lie operator to first order from a map point of view [6]. Even though both methods are mathematically equivalent and straightforward to first order, the latter approach is significantly simpler if higher order calculations are needed. In the following we illustrate the theory in the case of linearized equations of motion and, implicitly how it has been generalized to the non-linear case.

The linear betatron motion is described by the Hamiltonian [7]

$$
H_{1}\left(x_{i}, p_{x i} ; s\right)=\frac{p_{x i}^{2}}{2}+K_{x}(s) \frac{x_{i}^{2}}{2}+\frac{q_{i}}{p_{0} c} \Phi\left(x_{i} ; s\right) \equiv H_{0}(s)+V(s)
$$

where $V(s)$ will be treated as a perturbation. Hamilton's equations leads to the well known Hill's equation

$$
x_{i}^{\prime \prime}+K_{x}(s) x_{i}=-\frac{q_{i}}{p_{0} c} \frac{\partial \Phi(s)}{\partial x_{i}} .
$$

The action-angle variables $\left(J_{x i}, \phi_{x i}\right)$ for the unperturbed motion are

$$
\begin{aligned}
x_{i}(s) & =\sqrt{2 J_{x i} \beta_{x}(s)} \cos \phi_{x i}(s), \\
p_{x i}(s) & =-\sqrt{\frac{2 J_{x i}}{\beta_{x}(s)}}\left[\sin \phi_{x i}(s)+\alpha_{x}(s) \cos \phi_{x i}(s)\right]
\end{aligned}
$$


with the corresponding Hamiltonian

$$
H_{2}\left(J_{x i}, \phi_{x i} ; s\right)=\frac{J_{x i}}{\beta_{x}(s)}+\frac{q_{i}}{p_{0} c} \Phi\left(\sqrt{2 J_{x i} \beta_{x}(s)} \cos \phi_{x i} ; s\right) .
$$

The unperturbed Hamilton's equations are

$$
J_{x i}^{\prime}=-\frac{\partial H_{0}}{\partial \phi_{x i}}=0, \quad \phi_{x i}^{\prime}=\frac{\partial H_{0}}{\partial J_{x i}}=\frac{1}{\beta_{x}(s)}
$$

so that $J$ is a constant of motion. Integrating the equations of motion leads to the well known definition of phase advance

$$
\begin{aligned}
& J_{x i}(s)=J_{0 x i} \\
& \phi_{x i}(s)=\int_{s_{0}}^{s} \frac{d u}{\beta_{x}(u)}+\phi_{0 x i} \equiv \mu_{x}(s)+\phi_{0 x i}
\end{aligned}
$$

and tune

$$
\nu_{x} \equiv \frac{1}{2 \pi} \oint \frac{d s}{\beta_{x}(s)}
$$

Averaging over one turn leads to the averaged Hamiltonian

$$
\left\langle H_{2}\left(J_{x i}, \phi_{x i} ; s\right)\right\rangle=\left\langle\frac{1}{\beta_{x}(s)}\right\rangle J_{x i}+\frac{q_{i}}{p_{0} c}\langle\Phi(s)\rangle
$$

where

$$
\begin{aligned}
\frac{1}{\bar{\beta}_{x}} & \equiv\left\langle\frac{1}{\beta_{x}(s)}\right\rangle=\frac{1}{C} \int_{s_{0}}^{s_{0}+C} \frac{d s}{\beta_{x}(s)}=\frac{2 \pi \nu_{x}}{C} \equiv \frac{\omega_{\beta x 0}}{C}, \\
\langle\Phi(s)\rangle\left(J_{x i}, \phi_{x i}\right) & =\frac{1}{C} \int_{s_{0}}^{s_{0}+C} \Phi\left(J_{x i}, \phi_{x i} ; s\right) d s
\end{aligned}
$$

and $\left(J_{x i}, \phi_{x i}\right)$ are given by eq. (6). The averaged equation of motion is then

$$
x_{i}^{\prime \prime}+\frac{\omega_{\beta x 0}^{2}}{c^{2}} x_{i}=\frac{q_{i}}{p_{0} c} \frac{\partial\langle\Phi(s)\rangle}{\partial x_{i}}
$$

averaging the potential rather than the force. 


\subsection{Wake Fields and Wake Potentials}

In this section we will introduce the concept of wake functions and wake potentials. We will do this in some detail for two reasons. The first is that we found exsisting litterature to be somewhat confusing, e.g. different conventions used even by the same author in several cases. The second reason is that the wake potentials are normally defined for straight line motion or harmonic time dependence. This will in the next section be generalized to the betatron motion, without assuming the beta function to be constant along the lattice as is customarily assumed.

We found Chao's treatment of the definition of wake potentials [8] very clear, but noted a change of sign conventions from an earlier publication [3]. This section closely follows his later treatment. However, we will use the following slightly modified conventions and definitions, since they lead to a formalism more transparent for generalizing to the case of e.g. varying beta function along the lattice.

The wake function is defined so that $W(\Delta s)=0, \Delta s<0$ as in Chao's earlier publication. This leads to an impedance defined by the complex conjugate of the "conventional" Fourier transform. In Chao's later publication, this is avoided by a change of convention: $W(\Delta s)=0, \Delta s>0$, but still leading to the same explicit formulas for e.g. impedances. However, in this case we find it more natural to let a beam current with time dependence $\exp (-i \omega t)$ define the complex conjugate of the impedance. This leads to more systematic conventions, in particular for cases where the beam current and the fields are general Fourier transforms. Note that this convention is compatible with Zotter's [9], where the impedance is defined from a beam current and corresponding fields with harmonic time evolution. The mentioned differences are easily confirmed by a quick inspection of the explicit formulas for e.g. resistive wall in the references $[3,8,9,10]$.

The wake function is defined with respect to the wake potential per unit length, as in the earlier work by Chao and later by Bane [11], rather than the total wake potential later used by Chao and earlier by Bane et al. [5]. This allows for a systematic distinction between impedance per unit length and total impedance, which will clearify the general formalism. Note also that we make a fundamental distinction between wake potential and wake function, the latter being the corresponding Green function for the potential problem. In addition we have as Chao taken care to avoid incorrect notations 
like a scalar on the L.H.S. of an equal sign and a vector on the R.H.S. (!), something that appears more than once in the existing litterature.

The Lorentz force due to a wake field, excited by a particle with charge $q_{j}$ moving with velocity $v$, on a trailing test particle separated by $\Delta s=s^{\prime}-s=$ $v t-s$ and with charge $q_{i}$ is given by

$$
\vec{F}(\Delta s, s)=q_{i}\left[\vec{E}_{\text {wake }}(\Delta s, s)+\vec{v} \times \vec{B}_{\text {wake }}(\Delta s, s)\right], \quad \Delta s \geq 0 .
$$

Note that the wake fields are in general functions of the space-time coordinates of both the exciting charge and the point of evaluation. This general but complicated description of wake fields can be dramatically simplified by introducing wake potentials $[3,8,5,12]$ obtained by averaging the force along a structure. In the case of a ring, this leads in general to a perturbative treatment since the motion of the charge has to be known (compare with the Born approximation in quantum mechanics). This treatment can be further simplified by neglecting the velocity dependence of the wake field by assuming the particles to move with the speed of light $c$.

In cylindrical coordinates one finds for the Lorentz force

$$
\begin{aligned}
& F_{\|}=q_{i} E_{s}, \\
& \vec{F}_{\perp}=q_{i}\left[\hat{\theta}\left(E_{\theta}+c B_{r}\right)+\hat{r}\left(E_{r}-c B_{\theta}\right)\right]
\end{aligned}
$$

which have to be simultaniously solved with Maxwell's equations

$$
\begin{aligned}
\frac{1}{r} \frac{\partial\left(r E_{r}\right)}{\partial r}+\frac{1}{r} \frac{\partial E_{\theta}}{\partial \theta}+\frac{\partial E_{s}}{\partial s} & =\frac{\rho}{\varepsilon_{0}} \\
\frac{1}{r} \frac{\partial B_{s}}{\partial \theta}-\frac{\partial B_{\theta}}{\partial s} & =\mu_{0} j_{r}+\frac{1}{c^{2}} \frac{\partial E_{r}}{\partial t} \\
\frac{\partial B_{r}}{\partial s}-\frac{\partial B_{s}}{\partial r} & =\mu_{0} j_{\theta}+\frac{1}{c^{2}} \frac{\partial E_{\theta}}{\partial t} \\
\frac{1}{r} \frac{\partial\left(r B_{\theta}\right)}{\partial r}-\frac{1}{r} \frac{\partial B_{r}}{\partial \theta} & =\mu_{0} j_{s}+\frac{1}{c^{2}} \frac{\partial E_{s}}{\partial t} \\
\frac{1}{r} \frac{\partial\left(r B_{r}\right)}{\partial r}+\frac{1}{r} \frac{\partial B_{\theta}}{\partial \theta}+\frac{\partial B_{s}}{\partial s} & =0 \\
\frac{1}{r} \frac{\partial E_{s}}{\partial \theta}-\frac{\partial E_{\theta}}{\partial s}+\frac{\partial B_{r}}{\partial t} & =0 \\
\frac{\partial E_{r}}{\partial s}-\frac{\partial E_{s}}{\partial r}+\frac{\partial B_{\theta}}{\partial t} & =0 \\
\frac{1}{r} \frac{\partial\left(r E_{\theta}\right)}{\partial r}-\frac{1}{r} \frac{\partial E_{r}}{\partial \theta}+\frac{\partial B_{s}}{\partial t} & =0 .
\end{aligned}
$$


The average force is defined by

$$
\left.\langle\vec{F}(r, \theta, \Delta s)\rangle \equiv \frac{1}{L} \int_{0}^{L} \vec{F}(\bar{r}, t)\right|_{t=(s+\Delta s) / c} d s
$$

where $L$ is large compared to the length of the wall structure. Similar expressions holds for $\langle\vec{E}\rangle$ and $\langle\vec{B}\rangle$. The multipole expansion for a charge density $\rho(r, \theta)$ moving with the speed of light along $s$ is defined by

$$
\begin{aligned}
\rho(r, \theta) & =\sum_{m=0}^{\infty} \rho_{m}(r, \theta), \\
& =\sum_{m=0}^{\infty} \frac{2 I_{m}(s-c t)}{1+\delta_{m 0}} \lambda(s-c t) n(r) \cos m \theta
\end{aligned}
$$

where $I_{m}$ is the $m$ th moment of the beam defined by

$$
I_{m} \equiv \int_{0}^{\infty} \int_{0}^{2 \pi} \rho(r, \theta) r^{m+1} \cos m \theta d r d \theta
$$

and normalized so that

$$
\int_{0}^{L} \lambda(s) d s=N, \quad \int_{0}^{\infty} 2 \pi r^{m+1} n(r) d r=1
$$

where $\mathrm{N}$ is the number of particles. In the case of an infinitesimaly thin ring charge displaced by $a$ following a straight line along $s$

$$
\rho_{m}(r, \theta)=\frac{I_{m}}{\pi a^{m+1}\left(1+\delta_{m 0}\right)} \delta(s-c t) \delta(r-a) \cos m \theta
$$

with a total charge of $Q_{j}=I_{0}$. The current density inside the structure is

$$
j_{r}=j_{\theta}=0, \quad j_{s}=c \rho
$$

and it follows for example that

$$
\begin{aligned}
\frac{\partial\left\langle F_{r}(r, \theta, \Delta s)\right\rangle}{\partial \Delta s} & =\left.\frac{\partial}{\partial \Delta s} \frac{1}{L} \int_{0}^{L} F_{r}(\bar{r}, t)\right|_{t=(s+\Delta s) / c} d s \\
& =-\left.\frac{q_{i}}{L} \int_{0}^{L}\left(\frac{1}{c} \frac{\partial E_{r}}{\partial t}-\frac{\partial B_{\theta}}{\partial t}\right)\right|_{t=(s+\Delta s) / c} d s \\
& =-\left.\frac{q_{i}}{L} \int_{0}^{L}\left(\frac{c}{r} \frac{\partial B_{s}}{\partial \theta}-c \frac{\partial B_{\theta}}{\partial s}-\frac{\partial B_{\theta}}{\partial t}\right)\right|_{t=(s+\Delta s) / c} d s \\
& =-\frac{q_{i} c}{r}\left\langle\frac{\partial B_{s}}{\partial \theta}\right\rangle
\end{aligned}
$$


and the system of equations, eqs. $(12,13)$ becomes remarkably simple $[3,8]$

$$
\begin{aligned}
-\frac{q_{i} c}{r} \frac{\partial\left\langle B_{s}\right\rangle}{\partial \theta} & =-\frac{\partial\left\langle F_{r}\right\rangle}{\partial \Delta s}=\frac{\partial\left\langle F_{\|}\right\rangle}{\partial r}, \\
q_{i} c \frac{\partial\left\langle B_{s}\right\rangle}{\partial r} & =-\frac{\partial\left\langle F_{\theta}\right\rangle}{\partial \Delta s}=\frac{1}{r} \frac{\partial\left\langle F_{\|}\right\rangle}{\partial \theta}, \\
\frac{\partial\left(r\left\langle F_{r}\right\rangle\right)}{\partial r} & =-\frac{\partial\left\langle F_{\theta}\right\rangle}{\partial \theta}, \\
\frac{\partial\left(r\left\langle F_{\theta}\right\rangle\right)}{\partial r} & =\frac{\partial\left\langle F_{r}\right\rangle}{\partial \theta} .
\end{aligned}
$$

It follows that the averaged longitudinal and transverse wake forces are related by

$$
\nabla_{\perp}\left\langle F_{\| 1}\right\rangle=-\frac{\partial}{\partial \Delta s}\left\langle\vec{F}_{\perp}\right\rangle
$$

also known as the Panofsky-Wenzel theorem [13]. The solutions for a structure with axial symmetry are therefore of the form $[3,8]$

$$
\begin{aligned}
-\frac{q_{i}}{L} \Phi_{\|}(r, \theta, \Delta s) & \equiv-\left\langle F_{\|}(r, \theta, \Delta s)\right\rangle=q_{i} I_{m} \mathcal{W}_{m}^{\prime}(\Delta s) r^{m} \cos m \theta \\
\frac{q_{i}}{L} \vec{\Phi}_{\perp}(r, \theta, \Delta s) & \equiv\left\langle\vec{F}_{\perp}(r, \theta, \Delta s)\right\rangle \\
& =c_{i} I_{m} \mathcal{W}_{m}(\Delta s) m r^{m-1}(\hat{r} \cos m \theta-\hat{\theta} \sin m \theta) \\
q_{i} c\left\langle B_{s}(r, \theta, \Delta s)\right\rangle & =q_{i} I_{m} \mathcal{W}_{m}^{\prime}(\Delta s) r^{m} \sin m \theta
\end{aligned}
$$

where $\mathcal{W}_{m}(\Delta s)$ and its derivative are the transverse and longitudinal wake functions whereas $\Phi_{\|}(r, \theta, \Delta s)$ and $\vec{\Phi}_{\perp}(r, \theta, \Delta s)$ are the corresponding longitudinal and transverse wake potentials in the case of straight line motion. Causality implies that

$$
\mathcal{W}_{m}(\Delta s)=0, \quad \Delta s \leq 0 .
$$

Note that the wake function is simply the Green function for a given structure, e.g. the transverse wake potential due to an arbitrary beam current

$$
J_{m}(s, t)=c I_{m}(s, t)
$$


is given by superposition

$$
\begin{aligned}
\vec{\Phi}_{\perp}(s, t) / L= & \frac{1}{c} \int_{s}^{\infty} \mathcal{W}\left(s^{\prime}-s\right) J_{m}\left(s, t-\frac{s^{\prime}-s}{c}\right) d s^{\prime} \\
& \times m r^{m-1}(\hat{r} \cos m \theta-\hat{\theta} \sin m \theta) \\
= & \frac{1}{c} \int_{0}^{\infty} \mathcal{W}(\Delta s) J_{m}\left(s, t-\frac{\Delta s}{c}\right) d \Delta s \\
& \times m r^{m-1}(\hat{r} \cos m \theta-\hat{\theta} \sin m \theta)
\end{aligned}
$$

since

$$
t \rightarrow t-\frac{s^{\prime}-s}{c} .
$$

Note that in the general case the moments also have skew components so that

$$
\begin{aligned}
I_{m, x}-i I_{m, y} & \equiv \int_{0}^{\infty} \int_{0}^{2 \pi} \rho(r, \theta) r^{m+1} e^{-i m \theta} d r d \theta \\
& =\int_{-\infty}^{\infty} \int_{-\infty}^{\infty} \rho(x, y)(x-i y)^{m} d x d y
\end{aligned}
$$

\subsection{Transverse Equation of Motion with Wake Fields}

The transverse dipole wake function $\mathcal{W}_{1}(\Delta s)$ is the leading order contribution to the average transverse Lorentz force due to a transverse wake field excited by a macro particle with charge $Q_{j}$ on a trailing particle separated by $\Delta s$ and with charge $q_{i}$

$$
\begin{aligned}
\left\langle\vec{F}_{\perp}(\Delta s ; s)\right\rangle= & q_{i} Q_{j}\left(\left\langle x_{j}(s)\right\rangle \widehat{x}+\left\langle y_{j}(s)\right\rangle \widehat{y}\right) \mathcal{W}_{1}(\Delta s ; s) \\
& +O(2), \quad \Delta s>0
\end{aligned}
$$

where $s$ is used as independent variable as customary for the transverse dynamics. Note that we allow for parametric variation of the wake function with $s$. The unit of $\mathcal{W}_{1}(\Delta s ; s)$ is $\mathrm{V} \mathrm{C}^{-1} \mathrm{~m}^{-2}$ in the SI system.

A consistent averaging of the equations of motion is straightforward using a Hamiltonian formulation, i.e. averaging of the perturbation expressed in action-angle variables for the unperturbed Hamiltonian. In fact, the Hamiltonian formalism was developed for this purpose in celestial mechanics. A 
Hamiltonian formulation is possible (i.e. relativistic interacting particles) since only the effect of a given wake field on other particles is considered, neglecting the individual particles effect on the wake field (i.e. external field approximation) [2]. It follows that

$$
\begin{aligned}
H_{1}\left(x_{i}, p_{x i} ; s\right)= & \frac{p_{x i}^{2}}{2}+K_{x}(s) \frac{x_{i}^{2}}{2}-\frac{q_{i} Q_{j}}{p_{0} c} x_{i} \sum_{n=0}^{N} \mathcal{W}_{1}\left(\Delta s_{i \rightarrow j}+n C ; s\right) \\
& \times\left\langle x_{j}(s-n C)\right\rangle
\end{aligned}
$$

where $\Delta s_{i \rightarrow j}=s_{i}-s_{j}$. The averaged Hamiltonian is obtained by transforming to action-angle variables and averaging over one turn

$$
\begin{aligned}
\left\langle H_{2}\left(J_{x}, \phi_{x}\right)\right\rangle= & \frac{\omega_{\beta x 0}}{c} J_{x i}-\left\langle\frac{2 q_{i} Q_{j}}{p_{0} c} \beta_{x}(s) \sqrt{J_{x i}\left\langle J_{x j}\right\rangle} \cos \phi_{x i}(s)\right. \\
& \left.\times \sum_{n=0}^{N} \mathcal{W}_{1}\left(\Delta s_{i \rightarrow j}+n C ; s\right) \cos \left\langle\phi_{x j}(s-n C)\right\rangle\right\rangle \\
= & \frac{\omega_{\beta x 0}}{c} J_{x i}-\frac{q_{i} Q_{j}}{p_{0} c C} \bar{\beta}_{x} \sqrt{J_{x i}\left(J_{x j}\right\rangle} \sum_{n=0}^{N} W_{1}\left(\Delta s_{i \rightarrow j}+n C\right) \\
& \times \cos \left(\phi_{0 x i}-\left\langle\phi_{0 x j}\right\rangle+n 2 \pi \nu_{x}\right)
\end{aligned}
$$

so that the effective transverse wake function is given by

$$
W_{1}(\Delta s) \equiv \frac{1}{\bar{\beta}_{x}} \oint \beta_{x}(s) \mathcal{W}_{1}(\Delta s ; s) d s \quad\left[\frac{\mathrm{V}}{\mathrm{Cm}}\right]
$$

This result differs from e.g. ref. [14] where the analysis is only valid in the limit the beta function does not vary along the structure.

The corresponding initial Hamiltonian, with the effect of the wake field averaged and lumped into a single kick at some location in the lattice, is then given by

$$
\begin{aligned}
H_{3}\left(x_{i}, p_{x i} ; s\right)= & \frac{p_{x i}^{2}}{2}+K_{x}(s) \frac{x_{i}^{2}}{2}-\frac{q_{i} Q_{j} \bar{\beta}_{x}}{p_{0} c \beta_{x 0}} x_{i} \sum_{n=0}^{N} W_{1}\left(\Delta s_{i \rightarrow j}+n C\right) \\
& \times\left\langle x_{j}(s-n C)\right\rangle \delta_{p}\left(s-s_{0}\right)
\end{aligned}
$$

where

$$
\delta_{p}\left(s-s_{0}\right) \equiv \sum_{n=0}^{\infty} \delta\left(s-s_{0}-n C\right)
$$


The corresponding Hamilton's equations are

$$
\begin{aligned}
x^{\prime}= & \frac{\partial H_{3}}{\partial p_{x}}=p_{x} \\
p_{x}^{\prime}= & -\frac{\partial H_{3}}{\partial x}=K_{x}(s) x_{i}+\frac{q_{i} Q_{j} \bar{\beta}_{x}}{p_{0} c \beta_{x 0}} \sum_{n=0}^{N} W_{1}\left(\Delta s_{i \rightarrow j}+n C\right) \\
& \times\left\langle x_{j}(s-n C)\right\rangle \delta_{p}\left(s-s_{0}\right) .
\end{aligned}
$$

\subsection{Equation of Motion for a Bunch}

In the case of a beam, one has to consider a distribution of particles with varying initial conditions. Higher order terms in the equation of motion will lead to a spread in betatron frequencies, contributing to Landau damping. This effect will for simplicity be neglected in the following treatment (i.e. we consider the worst case for growth rates). However, it can be included as a straightforward generalization.

The Hamiltonian for the bunch motion is obtained by integrating over the bunchs' charge distribution

$$
\begin{aligned}
H_{4}\left(x_{k}, p_{x k} ; s\right)= & \int_{-\infty}^{\infty}\left[\frac{p_{x i}{ }^{2}}{2}+K_{x}(s) \frac{x_{i}{ }^{2}}{2}-\frac{q_{i} Q_{j} \bar{\beta}_{x}}{p_{0} c \beta_{x 0}} x_{i}\right] \rho(x) d x \\
& \times \sum_{n=-\infty}^{\infty} W_{1}\left(\Delta s_{i \rightarrow j}+n C\right) \\
& \times\left\langle x_{j}(s-n C)\right\rangle \delta_{p}\left(s-s_{0}\right) .
\end{aligned}
$$

The Hamiltonian for the rigid bunch motion is obtained with the distribution

$$
\rho(x)=\delta(x-\langle x\rangle)
$$

leading to

$$
\begin{aligned}
H_{4}\left(x_{k}, p_{x k} ; s\right)= & \frac{\left\langle p_{x k}\right\rangle^{2}}{2}+K_{x}(s) \frac{\left\langle x_{k}\right\rangle^{2}}{2}-\frac{Q_{k} Q_{j} \bar{\beta}_{x}}{p_{0} c \beta_{x 0}}\left\langle x_{k}\right\rangle \\
& \times \sum_{n=-\infty}^{\infty} W_{1}\left(\Delta s_{k \rightarrow j}+n C\right) \\
& \times\left\langle x_{j}(s-n C)\right\rangle \delta_{p}\left(s-s_{0}\right)
\end{aligned}
$$


when $N$ goes to infinity. Note that the sum can be extended to $-\infty$ since the wake potential is causal. Also note that in this case the wakefield is excited by the center of charge for bunch $j$. The corresponding Hamilton's equations are

$$
\begin{aligned}
\left\langle x_{k}\right\rangle^{\prime}= & \frac{\partial H_{4}}{\partial\left\langle p_{x k}\right\rangle}=\left\langle p_{x k}\right\rangle, \\
\left\langle p_{x k}\right\rangle^{\prime}= & -\frac{\partial H_{4}}{\partial\left\langle x_{k}\right\rangle} \\
= & -K_{x}(s)\left\langle x_{k}\right\rangle+\frac{Q_{k} Q_{j} \bar{\beta}_{x}}{p_{0} c \beta_{x 0}} \sum_{n=-\infty}^{\infty} W_{1}\left(\Delta s_{k \rightarrow j}+n C\right) \\
& \times\left\langle x_{j}(s-n C)\right\rangle \delta_{p}\left(s-s_{0}\right) .
\end{aligned}
$$

It should be realized that Hamilton's equations allow for a straightforward application of one turn maps for the lattice, e.g. its power series representation. This can easily be generalized to the non-linear case by considering a map that includes the necessary non-linear dynamics to faithfully represent the lattice. However, one has to keep in mind that a power series map has a finite radius of convergence and is not symplectic. Calibration of the model, e.g. determination of limits for betatron amplitudes and number of turns against a symplectic integrator (i.e. tracking code) should therefore as always be carried out. Alternatively, for more flexibility, one may consider using a fitted generating function (i.e. a fitted map) since such a map may be tailored to a certain area of phase space. The map is still approximate but has the additional advantage of being symplectic.

From the previous analysis the effect of the wake field has been reduced to a single local kick, easy to implement in any standard tracking code. Note however that since each excitation is acting over several turns, each individual excitation has to be kept and applied for a sufficient number of turns. This applies in particular to the resistive wall case, since the field is only damped as $1 / \sqrt{\Delta s}$. To avoid this complication, which dramatically reduces the efficiency in the case of many bunches, one may consider Fourier transforming and only keep track of a sufficient number of harmonics of the total wake field. However, such an approach breaks down since frequencies in a neighborhood of the harmonics also significantly contribute to the dynamics [15]. This is particularly apparent in the derivation of the Poisson sum formula [16], used in the following to introduce the impedance in the dispersion relations. 


\subsection{Transverse Impedance}

In the case of a beam with a current of the form

$$
J_{m}(s, t)=\widehat{J}_{m} e^{i(k s-\omega t)}
$$

where * stands for complex conjugate and $\widehat{J}_{m}$ is the Fourier transform of the current, the transverse wake potential is given by eq. (26)

$$
\begin{aligned}
\vec{\Phi}_{\perp} / L= & \frac{1}{c} m r^{m-1} \int_{0}^{\infty} \mathcal{W}_{m}(\Delta s) J_{m}\left(s, t-\frac{\Delta s}{c}\right) d \Delta s \\
& \times(\hat{r} \cos m \theta-\hat{\theta} \sin m \theta) \\
= & \frac{1}{c} m r^{m-1} \widehat{J}_{m} e^{i(k s-\omega t)} \int_{-\infty}^{\infty} \mathcal{W}_{m}(\Delta s) e^{i \omega \Delta s / c} d \Delta s \\
& \times(\widehat{r} \cos m \theta-\hat{\theta} \sin m \theta) \\
\equiv & m r^{m-1} \widehat{J}_{m} e^{i(k s-\omega t)} i \mathcal{Z}_{m}^{\perp \cdot}(\omega)(\hat{r} \cos m \theta-\hat{\theta} \sin m \theta) .
\end{aligned}
$$

The transverse local impedance (i.e. the transverse impedance per unit length) is therefore defined as the Fourier transform of the transverse wake function

$$
\mathcal{Z}_{m}^{\perp}(\omega ; s) \equiv \frac{i}{c} \int_{-\infty}^{\infty} \mathcal{W}_{m}(\Delta s ; s) e^{-i \omega \Delta s / c} d \Delta s \quad\left[\frac{\Omega}{\mathrm{m}^{2 m}}\right]
$$

Correspondingly, the transverse wake function is given by the inverse Fourier transform

$$
\mathcal{W}_{m}(\Delta s ; s)=\frac{1}{2 \pi i} \int_{-\infty}^{\infty} \mathcal{Z}_{m}^{\perp}(\omega ; s) e^{i \omega \Delta s / c} d \omega
$$

It is clear from eq. (42) that the transverse impedance has the following symmetry

$$
\mathcal{Z}_{m}^{\perp^{\bullet}}(\omega ; s)=-\mathcal{Z}_{m}^{\perp}(-\omega ; s)
$$

since the wake field is real. Furthermore, one can prove that eq. (22) implies the following relation between longitudinal and transverse local impedance

$$
\mathcal{Z}_{m}^{\|}(\omega)=\frac{\omega}{c} \mathcal{Z}_{m}^{\perp}(\omega), \quad m>0 .
$$


Alternatively the transverse local impedance may be defined as the transverse average force per unit charge along the beam trajectory divided by the moment of the beam

$$
\begin{aligned}
\mathcal{Z}_{m}^{\perp}(-\omega ; s) & =-\mathcal{Z}_{m}^{\perp^{*}}(\omega ; s) \\
& \equiv \frac{i \vec{\Phi}_{\perp} / L}{m r^{m-1} \widehat{J}_{m}^{*}(\omega, s) e^{i(k s-\omega t)}(\hat{r} \cos m \theta-\hat{\theta} \sin m \theta)} \\
& =\frac{i\left\langle[\vec{E}(\omega)+c \hat{s} \times \vec{B}(\omega)]_{\perp}^{*}\right\rangle}{m r^{m-1} \widehat{J}_{m}^{*}(\omega, s)(\widehat{r} \cos m \theta-\hat{\theta} \sin m \theta)}
\end{aligned}
$$

where we have Fourier expanded the fields.

Similarly, we define the total transverse impedance for the dipole case as the Fourier transform of the effective wake function

$$
\begin{aligned}
Z_{1}^{\perp}(\omega) & \equiv \frac{i}{c} \int_{-\infty}^{\infty} W_{1}(\Delta s) e^{-i \omega \Delta s / c} d \Delta s \\
& =\frac{1}{\bar{\beta}_{x}} \oint \beta_{x}(s) \mathcal{Z}_{\perp}(\omega ; s) d s \quad\left[\frac{\Omega}{\mathrm{m}}\right]
\end{aligned}
$$

Note that in the case of a general current rather than ring charges, it follows from eq. (41) that the corresponding transverse wake potential is given by the product of the local impedance and the Fourier transform of the current

$$
\vec{\Phi}_{\perp} / L=-\frac{1}{2 \pi} m r^{m-1} \int_{-\infty}^{\infty} \hat{J}_{m}(\omega) \mathcal{Z}_{m}^{\perp}(\omega ; s) e^{i \omega t} d \omega(\hat{r} \cos m \theta-\hat{\theta} \sin m \theta) .
$$

For example, in the case of resistive wall the wake potential for a point charge is $[3,17]$

$$
\mathcal{W}_{1}(\Delta s ; s)=\frac{\mathcal{W}_{1}(\Delta s=1 ; s)}{\sqrt{\Delta s}}, \quad \Delta s>0
$$

with the corresponding transverse local impedance

$$
\begin{aligned}
\mathcal{Z}_{1}^{\perp}(\omega ; s) & =\sqrt{\frac{\pi}{2 c}} \mathcal{W}_{1}(\Delta s=1 ; s) \frac{\operatorname{sgn}(\omega)+i}{\sqrt{|\omega|}} \\
& =\operatorname{Re}\left(\mathcal{Z}_{1}^{\perp}(\omega=1 ; s)\right) \frac{\operatorname{sgn}(\omega)+i}{\sqrt{|\omega|}}
\end{aligned}
$$




\subsection{Dispersion Relations}

The numerical simulations have been checked against analytical calculations in the case of a linear map for the lattice. In particular, the dispersion relation for initially displaced bunches (e.g. injected or kicked bunches) is obtained from the eigenmode ansatz

$$
\left\langle x_{k}\right\rangle=\left\langle x_{k 0}\right\rangle e^{-i \omega_{\beta x m} s / c}, \quad m=0,1,2, \cdots, M-1
$$

where $M$ is the number of bunches. Substitution into the averaged equation of motion with the wake potential distributed uniformly around the lattice

$$
\begin{aligned}
\left\langle x_{k}\right\rangle^{\prime \prime}+\frac{\omega_{\beta x 0}^{2}}{c^{2}}\left\langle x_{k}\right\rangle= & \frac{Q_{i} Q_{j}}{p_{0} C C} \sum_{j=1}^{M} \sum_{n=-\infty}^{\infty} W_{1}\left(\Delta s_{k \rightarrow j}+n C\right) \\
& \times\left\langle x_{j}(s-n C)\right\rangle
\end{aligned}
$$

leads to

$$
\begin{aligned}
\left(\omega_{\beta x m}^{2}-\omega_{\beta x 0}^{2}\right)\left\langle x_{k 0}\right\rangle= & -\frac{Q_{k} Q_{j} c}{p_{0} C}\left\langle x_{j 0}\right\rangle \sum_{j=1}^{M} \sum_{n=-\infty}^{\infty} W_{1}\left(\Delta s_{k \rightarrow j}+n C\right) \\
& \times e^{i\left(\Delta s_{k \rightarrow j}+n C\right) \omega_{\beta x m} / c}
\end{aligned}
$$

so that using

$$
\omega_{\beta x m}^{2}-\omega_{\beta x 0}^{2} \approx 2 \omega_{\beta x 0}\left(\omega_{\beta x m}-\omega_{\beta x 0}\right)
$$

one obtains

$$
\begin{aligned}
\omega_{\beta x m}\left\langle x_{k 0}\right\rangle= & \omega_{\beta x 0}\left\langle x_{k 0}\right\rangle-\frac{Q_{k} Q_{j} c}{2 p_{0} \omega_{\beta x 0} C}\left\langle x_{j 0}\right\rangle \sum_{j=1}^{M} \sum_{n=-\infty}^{\infty} W_{1}\left(\Delta s_{k \rightarrow j}+n C\right) \\
& \times e^{i\left(\Delta s_{k \rightarrow j}+n C\right) \omega_{\beta x m} / c}
\end{aligned}
$$

leading to the eigenvalue problem

$$
M\left\langle\bar{x}_{0}\right\rangle=\omega_{\beta x m}\left\langle\bar{x}_{0}\right\rangle
$$

with

$$
\begin{aligned}
M_{k j}= & \omega_{\beta x 0} \delta_{k j}-\frac{Q_{k} Q_{j} c}{2 p_{0} \omega_{\beta x 0} C} \sum_{n=-\infty}^{\infty} W_{1}\left(\Delta s_{k \rightarrow j}+n C\right) \\
& \times e^{i\left(\Delta s_{k \rightarrow j}+n C\right) \omega_{\beta x 0} / c}
\end{aligned}
$$


where $\omega_{\beta x m}$ has been approximated by $\omega_{\beta x 0}$ thereby implying a perturbative treatment. Solving the eigenvalue problem determines the eigenfrequencies.

Alternatively, the impedance is introduced by the Poisson sum formula [16]

$$
\sum_{n=-\infty}^{\infty} f(\alpha n)=\frac{1}{C} \sum_{p=-\infty}^{\infty} F\left(\frac{p 2 \pi}{\alpha}\right)
$$

with the Fourier transform defined by

$$
F(\omega) \equiv \int_{-\infty}^{\infty} f(t) e^{-i \omega t} d t
$$

and observing that

$$
f\left(t+t_{0}\right) \leftrightarrow F(\omega) e^{i \omega t_{0}}, \quad f(t) e^{i \omega_{0} t} \leftrightarrow F\left(\omega-\omega_{0}\right) .
$$

and it follows that

$$
M_{k j}=\omega_{\beta x 0} \delta_{k j}-\frac{i Q_{k} Q_{j} c^{2}}{2 p_{0} \omega_{\beta x 0} C^{2}} \sum_{p=-\infty}^{\infty} Z_{1}^{\perp}\left(p \omega_{0}-\omega_{\beta x 0}\right) e^{i p \omega_{0} \Delta s_{k \rightarrow j} / c}
$$

where

$$
\omega_{0} \equiv \frac{2 \pi}{T_{0}}=\frac{2 \pi c}{C} .
$$

The curresponding growth rate for each eigenmode is then given by

$$
\frac{1}{\tau_{m}}=\operatorname{Im}\left(\omega_{\beta x m}-\omega_{\beta x 0}\right)
$$

which is positive in the unstable case, with a tune shift

$$
\Delta \nu_{x m}=\operatorname{Re}\left(\omega_{\beta x m}-\omega_{\beta x 0}\right) .
$$

Note that in the case of resistive wall, the sum in eq. (61) for the frequency shift is not well defined and the limit $\Delta s$ is delicate [3].

A dispersion relation for initially non-displaced bunches (e.g. stored bunches) is obtained from the ansatz

$$
\left\langle x_{k}\right\rangle=\frac{s}{\tau c} e^{-i \omega_{\beta x 0} s / c} .
$$


Substitution into the averaged equation of motion determines the linear growth rate

$$
\frac{1}{\tau}=\frac{i Q_{k} Q_{j} c}{2 p_{0} \omega_{\beta x 0} C} \sum_{j=1}^{M} \sum_{n=-\infty}^{\infty} W_{1}\left(\Delta s_{k \rightarrow j}+n C\right)\left\langle x_{j 0}\right\rangle e^{i\left(\Delta s_{k \rightarrow j}+n C\right) \omega_{\beta \times 0} / c} .
$$

For completeness we would like to point out that the general initial value problem may be solved by Laplace transform [14].

\section{THE FACILITIES}

\subsection{The Advanced Light Source}

The ALS is a $1.5 \mathrm{GeV}$ Synchrotron Radiation Source. Tab. 1 and 2 summarize the parameters needed in our study [18]. The storage ring design is based on a triple bend achromat (TBA) lattice. Wigglers and undulators occupy a sizable part of the circumference. Theoretical and computational studies of their magnetic field effects on the beam dynamics has been done and are summarized in the conceptual report. The 11 straight sections for wigglers and undulators provide greatly enhanced proton-photon performance compared with bending magnets. A particular feature of the storage ring is the very small design value of the horizontal emittance of the electron beam, which has been minimized to maximize the spectral brightness of the undulator photon beams.

The injection system is illustrated in Fig. 1. It consists of a high-intensity electron gun, a $50 \mathrm{MeV}$ travelling wave linac, and a $1 \mathrm{~Hz}, 1.5 \mathrm{GeV}$ booster synchrotron. The injection system is designed to be able fill the storage ring to its design current of $400 \mathrm{~mA}$ in 2.1 minutes.

\subsection{Description of the Injection Process}

There are two different modes of operation for the storage ring, multibunch and few bunch mode. Each with special needs from the injection system [18]. The multibunch mode requires the storage ring to be filled to a current of $400 \mathrm{~mA}$ in approximately 250 consecutive bunches (out of 328 ). For the few-bunch mode, one expects a current of $7.6 \mathrm{~mA}$ per bunch and the bunches are filled one at a time. The beam transfer into and out of the booster are 


\begin{tabular}{|c|c|}
\hline Nominal energy $(\mathrm{GeV})$ & 1.5 \\
\hline Maximum circulating current, multibunch (mA) & 400 \\
\hline Number of stored electrons, multibunch & $1.6410^{12}$ \\
\hline Maximum circulating current, single bunch (mA) & 7.6 \\
\hline Number of stored electrons, single bunch & $3.1210^{10}$ \\
\hline Natural emittance (m.rad) & $4.0810^{-9}$ \\
\hline Natural energy spread, rms & $7.0110^{-4}$ \\
\hline Energy spread, rms, at max. current (multibunch) & $0.810^{-3}$ \\
\hline Energy spread, rms, at max. current (single bunch) & $1.4810^{-3}$ \\
\hline Bunch length, $\mathrm{rms}$, natural $(\mathrm{mm})$ & 3.7 \\
\hline Bunch length, $2 \sigma$, natural & $\overline{24}$ \\
\hline Bunch length, $2 \sigma$, maximum current (multibunch, $\mathrm{ps}$ ) & 28 \\
\hline Bunch length, $2 \sigma$, maximum current (single bunch, ps) & 47 \\
\hline $\begin{array}{ll}\text { Peak energy }(\mathrm{GeV}) \\
\end{array}$ & $\overline{1.9}$ \\
\hline Filling time, multibunch, to $400 \mathrm{~mA}(\mathrm{~min})$ & 2.1 \\
\hline Filling time, single bunch, to $7.6 \mathrm{~mA}$ per bunch $(\mathrm{s})$ & 16 \\
\hline Circumference (m) & $\overline{196.8}$ \\
\hline Orbital period (ns) & 656.4 \\
\hline Harmonic number & 328 \\
\hline Radio frequency(MHz) & 499.654 \\
\hline Peak effective rf voltage (MV) & 1.5 \\
\hline Number of superperiods & 12 \\
\hline Insertion straight section length $(\mathrm{m})$ & 6.75 \\
\hline Mean radius (m) & 31.32 \\
\hline Bending field $(T)$ & 1.248 \\
\hline Bending radius (m) & 4.01 \\
\hline Injection energy (GeV) & 1.5 \\
\hline Injection field $(\mathrm{T})$ & 1.248 \\
\hline
\end{tabular}

Table 1: ALS parameters. 


\begin{tabular}{|r|r|}
\hline Number of dipoles per superperiod & 3 \\
\hline Number of quadrupoles per superperiod & 6 \\
\hline Hor. betatron tune & 14.277 \\
\hline Vert. betatron tune & 8.179 \\
\hline Synchrotron tune & 0.0082 \\
\hline Hor. natural chromoticity & -24.1 \\
\hline Vert. natural chromaticity & -28.5 \\
\hline Hor. betatron function at insertion symmetry points (m) & 11.0 \\
\hline Vert. betatron function at insertion symmetry points (m) & 4.0 \\
\hline Hor. beam size at insertion symmetry points (rms) & $201 \mu m$ \\
\hline Vert. beam size at insertion symmetry points (rms) & 201 \\
$10 \%$ emittance ratio ( $\mu m)$ & \\
\hline Momentum compaction & $1.4310^{-9}$ \\
\hline Hor. damping time (ms) & 13.1 \\
\hline Vert. damping time (ms) & 17.6 \\
\hline Long. damping time (ms) & 10.7 \\
\hline 2 & 2 \\
\hline
\end{tabular}

Table 2: ALS lattice parameters. 


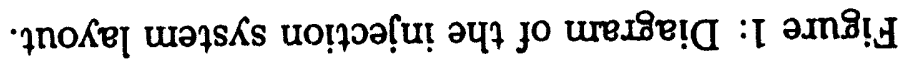
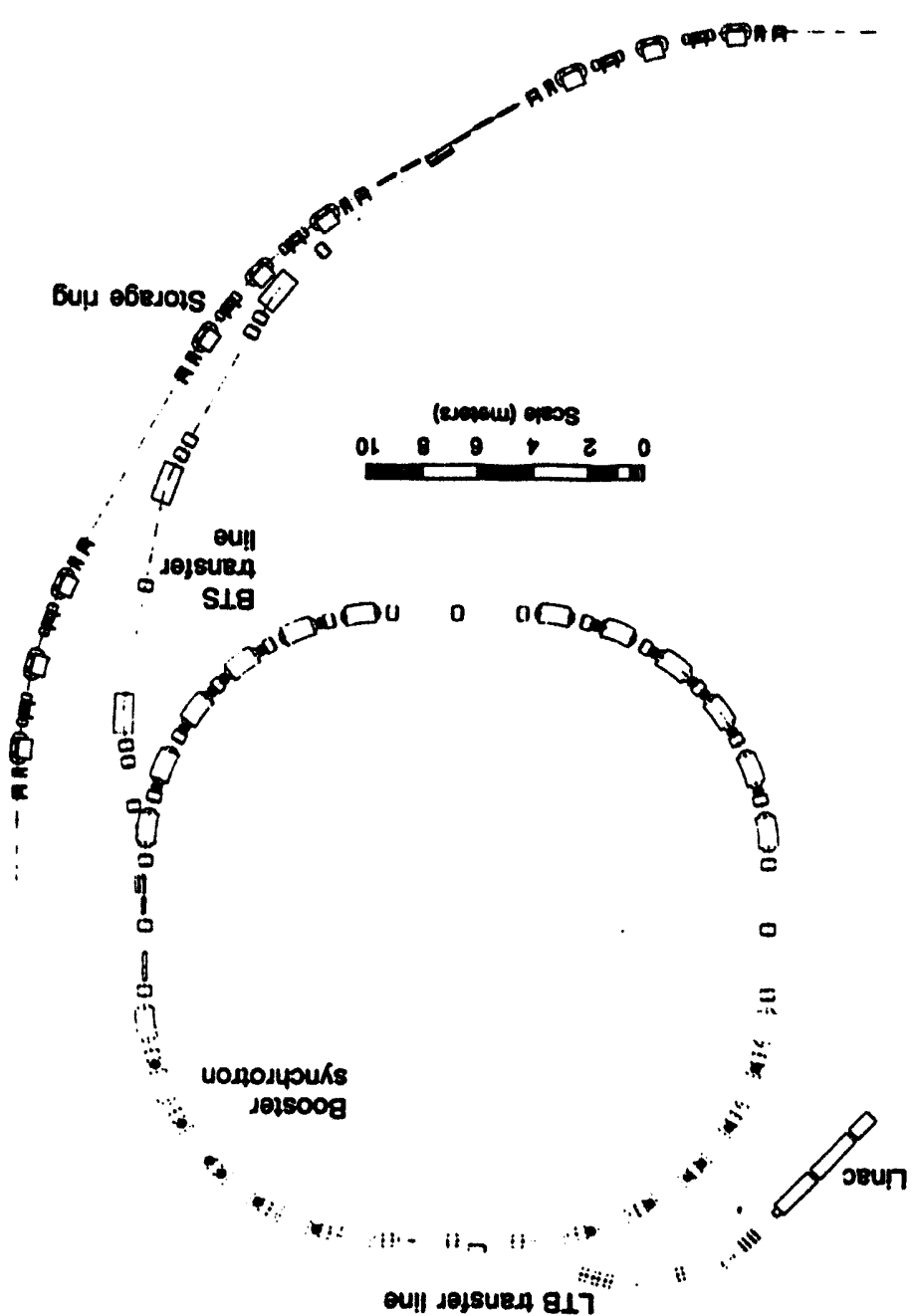
done in a single turn, using a full aperture fast kicker, requiring only a single kicker pulse per cycle.

In the multibunch mode, the electron gun is triggered with a long (about $100 \mathrm{~ns}$ ) pulse. Before entering the linac, the beam is chopped at $500 \mathrm{MHz}$ to minimize subsequent losses at higher energies. The beam from the linac is injected into the booster at $50 \mathrm{MeV}$ during a single turn, using of a septum magnet and a full aperture kicker. The incoming beam fills 50 (out of 125) $500-\mathrm{MHz}$ booster if buckets, is accelerated to $1.5 \mathrm{GeV}$ in the booster and extracted in a single turn, with a slow beam bump, a fast kicker, and two (thin and thick) septum magnets. By careful timing the storage ring is repetively filled with the required 250-bunch pattern.

In the few-bunch mode, the electron gun and linac are triggered to produce a single, short (about $0.5 \mathrm{~ns}$ ), high-intensity pulse, injected at $50 \mathrm{MeV}$ into a single booster of bucket. The single bunch is accelerated in the booster to $1.5 \mathrm{GeV}$ and extracted as before. The single bunch is transferred into a single rf bucket in the storage ring. The same storage ring bucket is filled repetitively, every booster cycle, until the desired current is reached.

Prior to injection, for both modes, a local bump is shifting the storage ring's closed orbit to the end of the injection septum, using four bump magnets as shown in Fig. 2. These magnets can displace the orbit up to $15 \mathrm{~mm}$. The beam is ejected from the booster and transported through the injection septa into the storage ring. These produce deflections of $8.0^{\circ}$ (thick) and $2.5^{\circ}$ (thin). The horizontal position of the thin injection septum is adjustable for best match to the dynamic aperture. Fig. 3 shows the position of the closed orbit and the injected beam, in the horizontal phase space, at the end of the septum magnet. The bump magnets are turned off in a time corresponding to about three revelution times to prevent the new beam from colliding with the septum. The injected beam is rapidly damped due to synchrotron radiation. The injection process is repeated at $1 \mathrm{~Hz}$ until the desired beam current is reached.

\section{THE TRACKING CODE}

The tracking code originates from a code developed at SLAC [19]. The code was restructured into independent subroutines, in order to allow for better portability. From two transverse dimensions $\left(x, p_{x}\right)$, we extended it to four 


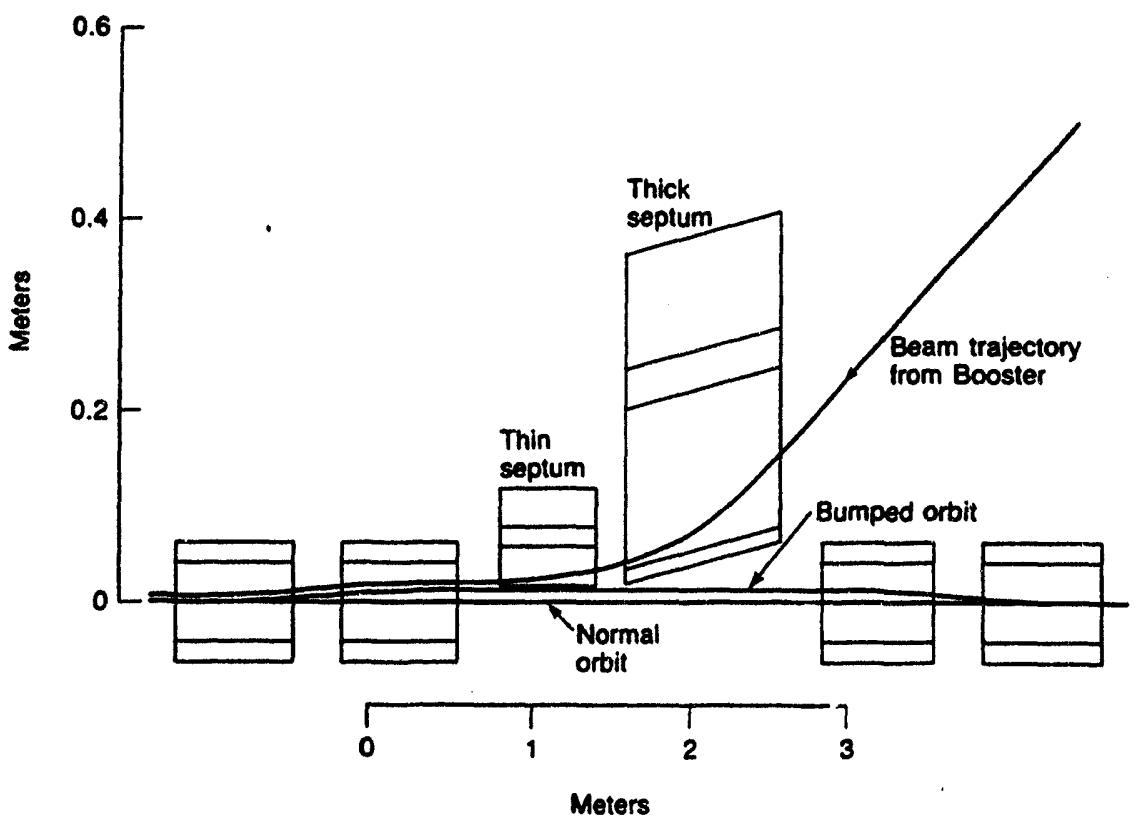

Figure 2: Diagram of the storage ring injection process.

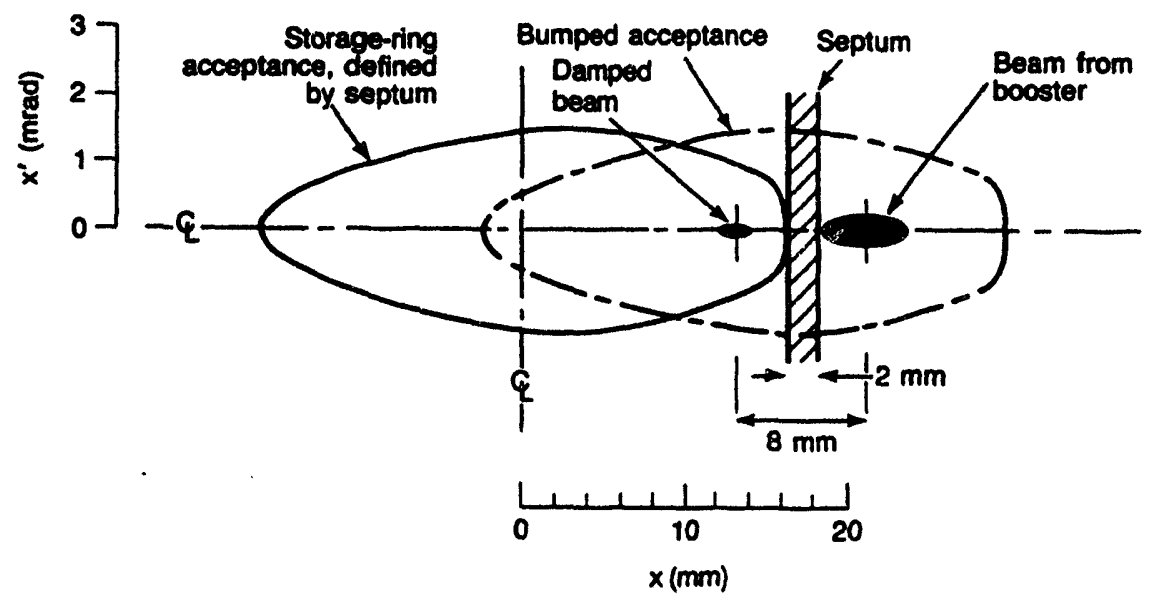

Figure 3: Storage ring acceptance, bumped acceptance, and the injected beam in horizontal phase space, just after the septum magnet. 
$\left(x, p_{x}, y, p_{y}\right)$, with the possibility to generalize to the full 6-dimensional case $\left(x, p_{x}, y, p_{y}, \delta, c t\right)$. The force due to the wake field is represented by a localized kick at one point in the lattice. The motion of a bunch at a given turn is divided into two parts. The first part describes the lattice by a one turn map, i.e. from just after the location of the wake kick all the way around the ring. The second part models the effect of the wake field by a thin kick in both transverse planes. The code allows the user to insert any map relevant to the lattice being studied. It may be a linear as well as a non-linear map expanded up to some arbitrary order $n$. The non-linear map is obtained from TRACY-2 [20] and DESPOT [21], i.e. the machine file is obtained from TRACY-2 and used as input file for DESPOT to extract the non-linear map for ALS. In the discussion of the results we will describe the importance of using a non-linear map to study the beam dynamics for ALS.

\section{THE CAVITY MODEL}

\subsection{The Radio Frequency System}

Tab. 3 summarizes the radio frequency parameters. Assuming that both rf cavities have been damped by a factor $\approx 30$ to a transverse shunt impedance of $2 \mathrm{M} \Omega / \mathrm{m}$ (maximum), we study the effect on the beam dynamics for HOMs with a shunt impedance larger than this value. The HOMs of the rf cavity have been measured [22] and Tab. 4 summarize the ones we used, either for program checking or beam dynamics studies.

\subsection{Cavity Wakefield and Computer Implementation}

The bunches of the $\mathrm{t}$ sam interact with each other through the electromagnetic fields they induce in the accelerator vacuum chamber. The effects of these fields, loosely referred to as wake fields, must therefore be included in the equations of motion.

In the case of an ultra-relativistic exciting particle of charge $q$ moving in the $s$ direction, the transverse wake potential per unit charge $\vec{\Phi}_{\perp}(\Delta s) / q$, is defined as the transverse momentum kick experienced by a trailing unit test 


\begin{tabular}{|r|r|}
\hline Number of cavities & 2 \\
\hline iris radius (m) & 0.035 \\
\hline cavity length (m) & 0.28 \\
\hline Frequency (MHz) & 499.654 \\
\hline Harmonic number & 328 \\
\hline Peak effective volatge, VT (MV) & 1.5 \\
\hline Transit time factor, T & 0.683 \\
\hline Total effective shunt impedance, ZT' $2(\mathrm{M} \Omega)$ & 16.0 \\
\hline Beam current, multibunch mode (mA) & 400 \\
\hline Power loss (kW): & \\
Synchrotron radiation, dipoles & 45 \\
Synchrotron radiation, insertion devices & 20 \\
Fundamental-mode dissipation & 140 \\
Parasitic modes & 2 \\
Waveguide and other losses & 20 \\
\hline Total rf power installed (kW) & 300 \\
\hline
\end{tabular}

Table 3: ALS radio frequency parameters.

\begin{tabular}{|c|c|c|c|}
\hline $\begin{array}{c}\text { Frequency } \\
{[\mathrm{MHz}]}\end{array}$ & Polarization & Quality factor & $\begin{array}{c}\text { Shunt impedance } \\
{[\mathrm{M} \Omega / \mathrm{m}]}\end{array}$ \\
\hline 810.08 & $\mathrm{H}$ & 48000 & 14.8 \\
\hline 1121.77 & $\mathrm{~V}$ & 7000 & 3.7 \\
\hline 1122.72 & $\mathrm{H}$ & 17000 & 9.0 \\
\hline 1801.61 & $\mathrm{~V}$ & 2000 & 1.1 \\
\hline
\end{tabular}

Table 4: Studied higher order cavity modes. 
charge $[4,8]$

$$
\vec{\Phi}_{\perp}(\Delta s) / q \equiv \frac{1}{q} \int_{0}^{C} d s[\vec{E}+\vec{v} \times \vec{B}]_{t=(s+\Delta s) / c}^{\perp}
$$

where $C$ is the circumference and where the unit of $\vec{\Phi}_{\perp}(\Delta s)$ is V/C. The accelerating structure is approximated by a periodic, symmetric disk-loaded structure with period $p$ and iris radius $b$. For an axially symmetric structure all the modes depend on the azimuthal angle $\theta$ as $\exp (\operatorname{im} \theta)$ where $m$ is an integer. The modes corresponding to $m=0,1,2$ are refered to as: longitudinal, dipole and quadrupole modes. In a periodic structure the modes appear in frequency bands. A charged particle travelling through the structure will excite a discrete set of these modes. Each mode $m n$ is characterized by a frequency $\omega_{m n} / 2 \pi$ (eigenvalue) and a modal loss factor $k_{m n}$ (eigenfunction). The modal loss factor for an infinitely short bunch is, using the "circuit definition" [4]

$$
k_{m n}=\frac{\omega_{m n} R_{m n}^{\|}}{2 Q_{m n}},
$$

with $R_{m n}^{\|}$the longitudinal shunt impedance in $\mathrm{Ohm}$ and $Q_{m n}$ the quality factor. The modal loss factor has the unit $\mathrm{L}^{-2 m-1}$. The coordinate system is arranged so that the transverse position of the exciting charge $r^{\prime}$ is in the horizontal plane, i.e. $\theta=0$. The test charge moves in the $s$ direction at a transverse position $\left(r=r^{\prime}, \theta\right)$ and a distance $\Delta s$ behind the exciting charge. The $m$-pole component of the wake potential per unit charge experienced by the test particle is a sum over all the $m$-pole modes $[5,11]$

$$
\begin{aligned}
\vec{\Phi}_{\perp}(\Delta s) / q= & m r^{\prime m} r^{m-1} \sum_{n=1}^{\infty} \frac{c R_{m n}^{\|} \omega_{m n}}{Q_{m n} \omega_{r}} \sin \left(\frac{\omega_{r} \Delta s}{c}\right) \exp \left(-\frac{\omega_{m n} \Delta s}{2 c Q_{m n}}\right) \\
& \times(\hat{r} \cos m \theta-\hat{\theta} \sin m \theta) \\
= & m r^{\prime m} r^{m-1} \sum_{n=1}^{\infty} \frac{2 c k_{m n}}{\omega_{r}} \sin \left(\frac{\omega_{r} \Delta s}{c}\right) \exp \left(-\frac{\omega_{m n} \Delta s}{2 c Q_{m n}}\right) \\
& \times(\hat{r} \cos m \theta-\hat{\theta} \sin m \theta), \quad \Delta s>0
\end{aligned}
$$

where

$$
\omega_{r} \equiv \omega_{m n} \sqrt{1-\frac{1}{4 Q_{m n}^{2}}}
$$


and note that

$$
\vec{\Phi}_{\perp}=0, \quad m=0 .
$$

The total wakefield is a sum over all the multipole contributions excited by the leading charge. Normally bunches remain near the axis and the diplacemenis $r^{\prime}$ and $r$ are small. The transverse wakefield is then dominated by the dipole modes and we can approximate the total wake potential by

$$
\begin{aligned}
\Phi_{x}(\Delta s) / q & \approx x \sum_{n=1}^{\infty} \frac{c R_{1 n}^{\|} \omega_{1 n}}{Q_{1 n} \omega_{r}} \sin \left(\frac{\omega_{r} \Delta s}{c}\right) \exp \left(-\frac{\omega_{1 n} \Delta s}{2 c Q_{1 n}}\right) \\
& \approx x \sum_{n=1}^{\infty} \frac{b^{2} \omega_{1 n} R_{1 n}^{\perp}}{Q_{1 n}} \sin \left(\frac{\omega_{r} \Delta s}{c}\right) \exp \left(-\frac{\omega_{1 n} \Delta s}{2 c Q_{1 n}}\right) \quad \Delta s>0(72)
\end{aligned}
$$

so that the wake potential is linear in the transverse displacement of the exciting charge. Note that the total wake function, i.e. the transverse wake potential per unit displacement and unit charge $W_{\perp}$ is given in units of $\mathrm{V} / \mathrm{pC} / \mathrm{m}$. The change of potential experienced by a unit test particle in Volts $\Phi_{\perp}$, is obtained by multiplying by the charge $q$ and the position $r^{\prime}$ of the exciting charge.

The code keeps track of the wake potential for each transverse dipole mode $n$. The potential increases just after a bunch passes through the cavity, since in the transverse case tr: bunch does not see its own local wake field. The wake potential advances in phase and attenuates (since $\omega_{1 n}$ is complex) between each bunch passage.

The complex transverse wake potential for mode $n$, just after bunch $j-1$ has passed through the cavity is denoted by $\Phi_{x}(j-1)$. The next bunch $j$ will experience the following complex wake potential from mode $n$ during its passage $[8,14]$

$$
\Phi_{x}(j)=\Phi_{x}(j-1) \exp \left(i \frac{\omega_{1 n} \Delta s_{j-1 \rightarrow j}}{c}\right) \exp \left(-\frac{\omega_{1 n} \Delta s}{2 c Q_{1 n}}\right)
$$

where $\omega_{1 n}$ is the frequency of the mode and $\Delta s_{j-1 \rightarrow j}$ is the longitudinal distance between bunch $j-1$ and $j$. Just after the passage of bunch $j$ the contribution to the exitation of mode $n$ is superimposed

$$
\Phi_{x}(j) \leftarrow \Phi_{x}(j)-i N_{j} e x_{j} W_{l n}
$$


since

$$
\sin \left(\frac{\omega_{1 n} \Delta s}{c}\right)=-\frac{i}{2}\left(e^{i \omega_{1 n} \Delta s / c}-e^{-i \omega_{1 n} \Delta s / c}\right)
$$

where $W_{1 n}$ is the effective wake function for mode $n$ in the expression of the transverse wake potential

$$
\Phi_{x}(\Delta s) / q=x W_{1 n} \sin \left(\frac{\omega_{1 n} \Delta s}{c}\right) \exp \left(-\frac{\omega_{1 n} \Delta s}{2 c Q_{1 n}}\right) .
$$

This is the contribution per cell and the unit of $W_{1 n}$ is $\mathrm{V} / \mathrm{C} / \mathrm{m}$. The real total change of transverse wake potential seen by bunch $j$ from all modes is

$$
\Phi_{x, \text { total }}(j)=\operatorname{Re} \sum_{n} \Phi_{x}(j)
$$

The formalism in the vertical plane is the same as the one shown for the horizontal plane.

\subsection{Dimensional Analysis}

The modal loss factor is

$$
k_{m n}=\frac{\omega_{m n} R_{m n}}{2 Q_{m n}}
$$

so that

$$
\left[k_{1 n}\right]=\left[\frac{\mathrm{V}}{\mathrm{TI}}\right]=\left[\frac{\mathrm{V}}{\mathrm{C}}\right]
$$

The transverse wake potential is

$$
\vec{\Phi}_{\perp}(\Delta s) / q=\vec{r} \sum_{n=0}^{\infty} W_{1 n} \sin \left(\frac{\omega_{1 n} s}{c}\right)
$$

with $\vec{r}$ the radial position $(\vec{r}=x \hat{x}+y \hat{y})$ and $W_{1 n}$ the effective wake function, i.e. the coefficient of the wake potential

$$
W_{1 n}=\frac{2 c k_{1 n}}{b^{2} \omega_{n}}
$$


and the dimension is

$$
W_{1 n}=\left[\frac{T L V}{L^{2} T C}\right]=\left[\frac{V}{C m}\right] .
$$

The unit of the wake potential is

$$
\left[\vec{\Phi}_{\perp}\right]=[V]
$$

as expected. Note that the transverse kick experienced by a unit test charge from the exciting charge $q$ is

$$
q r^{\prime} W_{1 n}
$$

with $r^{\prime}$ the displacement of the exciting charge.

\subsection{Growth Rate}

To test the program, we detuned the cavity onto the betatron sideband. For a single bunch we find for the growth rate

$$
\tau^{-1}=-\frac{N e^{2} c^{2} \beta_{c a v}}{2 E C^{2}} \sum_{p=-\infty}^{+\infty} \operatorname{Re}\left(Z_{1}^{\perp}\left(p \omega_{0}-\omega_{\beta}\right)\right)
$$

with $C$ the circumference and $c$ the speed of light. We assume that the beta function is constant within the cavity. In this case the impedance has a simple expression

$$
\tau^{-1}=-\frac{N e c^{2} \beta_{c a v}}{2 E / e C^{2}} R_{\perp}
$$

with $R_{\perp}$ the transverse shunt impedance.

We checked the code with a cavity mode detuned on the first lower sideband using a single macro particle $\left(5 \times 10^{9} \mathrm{part} / \mathrm{bunch}\right)$. The horizontal growth rate was estimated by tracking 4096 turns with an initial horizontal displacement of $5.0 \mathrm{~mm}$. The HOM at $810.08 \mathrm{MHz}$ was detuned to the lower sideband $f_{-}=809.993 \mathrm{MHz}$. The calculated growth rate is $9.65 \mathrm{msec}$ and we found $9.76 \mathrm{msec}$ from tracking. Similarly the HOM at $1122.72 \mathrm{MHz}$ was detuned to $f_{-}=1122.277 \mathrm{MHz}$. The calculated growth rate is $15.9 \mathrm{msec}$ and we found $16.0 \mathrm{msec}$ from tracking. The discrepancy in the first case is due to the fact that the dispersion relation only gives the first order perturbations. We proved this by a simple scaling test, i.e. we reduced the impedance to 10.0 $\mathrm{M} \Omega / \mathrm{m}$ and found $14.4 \mathrm{msec}$ from tracking compared to $14.3 \mathrm{msec}$ calculated. 


\begin{tabular}{|c|c|}
\hline $\begin{array}{c}\text { time constant } \\
\text { [msec] }\end{array}$ & $\begin{array}{c}\text { quality factor } \\
Q\end{array}$ \\
\hline 16.2 & 136000 \\
\hline 16.0 & 68000 \\
\hline 16.0 & 34000 \\
\hline 16.0 & 17000 \\
\hline 16.1 & 8500 \\
\hline 16.7 & 4250 \\
\hline 19.4 & 2125 \\
\hline
\end{tabular}

Table 5: Scaling of the quality factor.

\subsection{Filling Time}

The filling time is given by [12]

$$
T_{F}=\frac{2 Q}{\omega_{m}(1+\beta)}
$$

with $Q$ the quality factor and $\beta \approx 0$ the coupling factor. A numerical evaluation for the mode $1122.72 \mathrm{MHz}(Q=17000)$ gives a filling time of $4.8 \mu \mathrm{sec}$. As the period $T_{0}$ is $0.66 \mu \mathrm{sec}$, it means that the cavity reaches steady state after roughly 10 turns.

\subsection{Scaling of the Quality Factor}

For the same mode a scaling of $Q$ was done to observe the limit of the validity of the analytical growth rate formula versus the value of $Q$. As expected, we found that the time constant starts to increase when the quality factor becomes sufficiently small as shown in Tab. 5 .

We also addressed the possibility of overlaping sidebands. For the same mode (at $1122.72 \mathrm{MHz}$ and a quality factor of 17000 ) the bandwidth (given by $\left.\Delta \omega=\omega_{1 n} / 2 Q\right)$ is $33 \mathrm{kHz}$ compared to the distance between the two sidebands (given by $\Delta f=f_{+}-f_{-}$) is $844 \mathrm{kHz}$. In addition, to check for a possible overlap between the first upper $\left(f_{1+}\right)$ and the second lower sideband $\left(f_{2-}\right)$, we computed the distance between these two sidebands which is $\Delta f_{1+/ 2-}=679$ kHz. 


\section{THE RESISTIVE WALL PARAMETERS}

\subsection{Definition}

In the following we present a breif background of the formulas for the resistive wall effect following Zotter [9] in order to get some insight into the driving mechanism. The betatron motion of the beam drives a differential wall current $I_{w}$ that flows in opposite directions on either side of the vacuum chamber. This leads to a longitudinal electric field $E_{s}$ that varies in strength across the aperture, and a transverse magnet field $B_{x}$. The longitudinal electric field extracts energy from the beam that drives the wall currents. These excites a dipole magnetic field that deflects the beam. The beam current density is given by

$$
J_{0}\left(x+a e^{i \omega t}, y, s\right) \approx J_{0}(x, y, s)+\frac{\partial J_{0}}{\partial x} a e^{i \omega t}
$$

with the beam current

$$
I=\iint J_{0}(x, y, s) d x d y
$$

The fields in the median plane are

$$
E_{s}=-\frac{x E_{0}}{b} e^{i \omega t}, \quad B_{y}=\frac{i E_{0}}{\omega b} e^{i \omega t}
$$

since

$$
\nabla \times \vec{E}+\frac{\partial \vec{B}}{\partial t}=0
$$

The wall current $I_{w}$ is obtained from the power lost per unit length by the beam due to the $E_{s}$ field, which for Harmonic fields is

$$
P=\frac{1}{2} \iint \operatorname{Re}(\vec{J} \cdot \vec{E}) d x d y=-\frac{a E_{0}}{2 b} \iint x \frac{\partial J_{0}^{*}}{\partial x} d x d y=\frac{a E_{0} I^{*}}{2 b}
$$

which is equal to the power flow into walls

$$
P=-I_{w}^{*} E_{0}
$$


so that

$$
I_{w}=-\frac{a I}{2 b}
$$

The wall current is related to the electric field at the wall $E_{0}$ by the longitudinal wall impedance $Z_{0}^{\|}$. It can be shown that, in the case of a round pipe with a current distribution $\cos \theta$, by assuming $I_{w}$ to be concentrated in about 1/4 the pipe circumference on either side, one gets the correct result [8]. The effective impedance is therefore $4 Z_{0}^{\|}$and

$$
V=4 I_{w} Z_{0}^{\|}=C E_{0}
$$

or

$$
E_{0}=\frac{4 I_{w} Z_{0}^{\|}}{C}
$$

with a deflecting magnetic field

$$
B_{x}=-\frac{i 2 a Z_{0}^{\|} I}{b^{2} \omega C} e^{i \omega t}
$$

When this is inserted into the definition of $Z_{1}^{\perp}$

$$
Z_{1}^{\perp}(\omega)=\frac{i \int_{0}^{C}[\vec{E}(s, \omega)+c \hat{s} \times \vec{B}(s, \omega)]_{\perp} d s}{\widehat{J}_{1}(\omega)(\hat{r} \cos m \theta-\hat{\theta} \sin m \theta)} \quad\left[\frac{\Omega}{\mathrm{m}}\right]
$$

one finds

$$
Z_{1}^{\perp}=\frac{2 c Z_{0}^{\|}}{b^{2} \omega}
$$

It follows that any structure that allows for a varying longitudinal electric field across the aperture or correspondingly, the forming of wall current loops that produce a dipole magnet field, will increase $Z_{1}^{\perp}$.

The transverse coupling impedance of a smooth beam pipe of radius $b$ and resistivity $\rho$ is given as a function of the frequency $\omega$ by [8]

$$
Z_{1}^{\perp}(\omega)=\frac{c C}{\pi b^{3}} \sqrt{\frac{\mu_{0} \rho}{2|\omega|}}(\operatorname{sgn} \omega+i)
$$




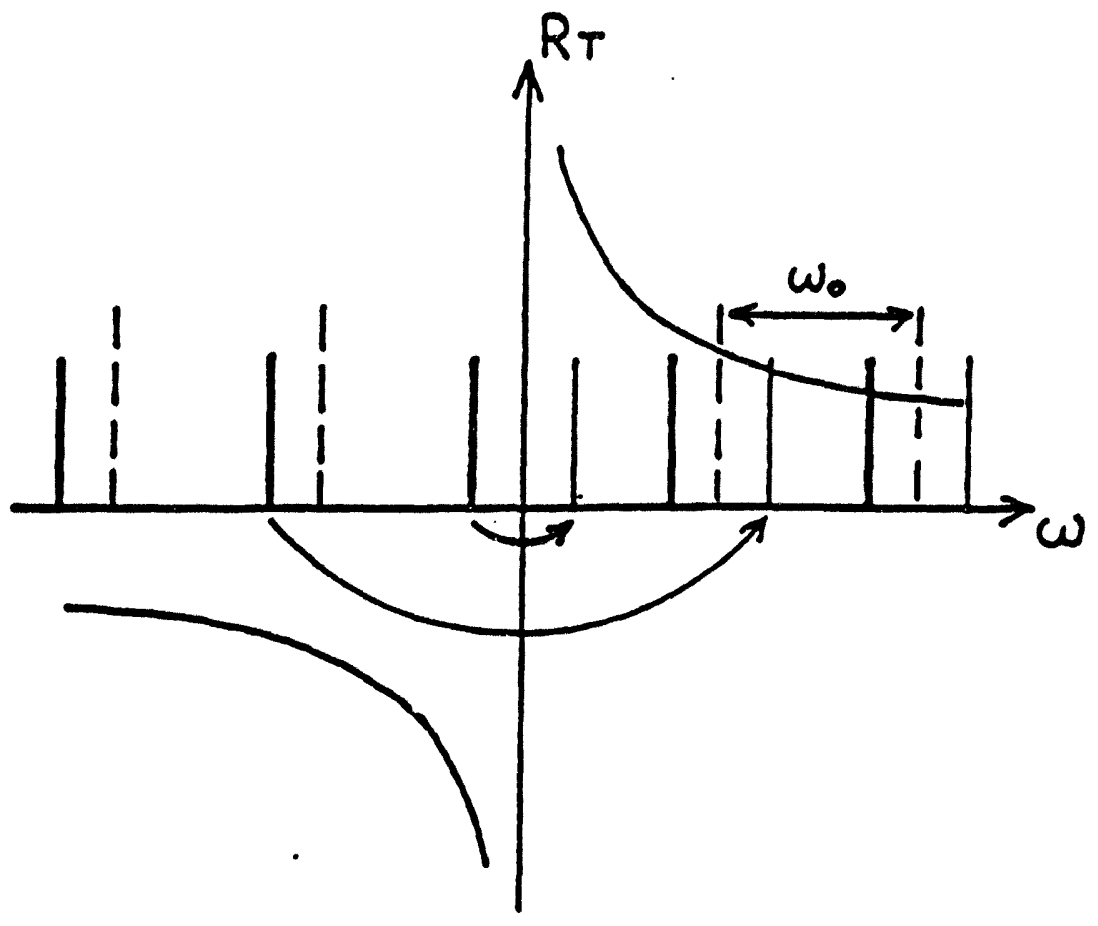

Figure 4: The resistive wall impedance as a function of frequency.

or

$$
\begin{aligned}
W_{1}(\Delta s) & =\sqrt{\frac{2 c}{\pi \Delta s}} \operatorname{Re}\left[Z_{1}^{\perp}(\omega=1)\right] \\
& =\sqrt{\frac{2 c \omega_{1}}{\pi \Delta s}} \operatorname{Re}\left[Z_{1}^{\perp}\left(\omega=\omega_{1}\right)\right]
\end{aligned}
$$

This function is illustrated in Fig. 4 together with the frequency lines of the coherent modes. If the tune is just below an integer, there is one slow wave close to the origin where the value of the resistive wall impedance is large, leading to a large growth rate of the instability. When this effect is dominant, it is preferable to choose a tune just above an integer, since this moves the frequency of the most dangerous mode to higher values and reduces its growth rate. 


\subsection{Resistive Wall Parameter for the ALS and Com- puter Implementation}

We used the following value for the ALS total impedances at the first sideband [23]: $0.355 \mathrm{M} \Omega / \mathrm{m}$ in the horizontal plane and $0.883 \mathrm{M} \Omega / \mathrm{m}$ in the vertical plane. The resistive wall subroutine bas been checked by studying the growth rate. The calculated growth rate is obtained from

$$
\begin{aligned}
\frac{1}{\tau} & =\operatorname{Im}\left(\omega_{\beta}-\omega_{0}\right) \\
& =-\frac{N e c^{3}}{2 E_{0} / e \omega_{\beta} C^{2}} \sum_{p=-\infty}^{+\infty} \operatorname{Re}\left(Z_{1}^{\perp}\left(p \omega_{0}-\omega_{\beta}\right)\right) \\
& =-\frac{N e c^{2}}{2 E_{0} / e \omega_{\beta} C} \sum_{n=1}^{\infty} \operatorname{Im}\left(W_{1}(n C) e^{i n \omega_{\beta} C / c}\right)
\end{aligned}
$$

By tracking a single macro particle and by only considering the resistive wall effect (no cavity), we obtain a horizontal growth time of $2.00 \mathrm{sec}$ and a vertical growth time of $0.627 \mathrm{sec}$. The calculated horizontal is $2.00 \mathrm{sec}$ and the calculated vertical is $0.625 \mathrm{sec}$, showing excellent agreement.

The computer implementation is based on the wake field. To be able to include effects due to the rather slow decay of the wake field in this case, we decided to keep track of the wakefield over several turns. After each turn, the new value of the wake field is shifted into a finite queue of preceding ones. The kick due to the wakefield is

$$
\begin{aligned}
\left\langle p_{x k}\right\rangle^{\prime}= & \frac{N e}{E_{0} / e} \sum_{n=-\infty}^{\infty} W_{1}\left(\Delta s_{k \rightarrow j}+n C\right) \\
& \times\left\langle x_{j}\left(s-\Delta s_{k \rightarrow j}-n C\right)\right\rangle \delta_{p}\left(s-s_{0}\right) .
\end{aligned}
$$

If we consider $a$ bunch $k$ on turn $n$, we have to consider three different contributions

- the kick on the current bunch from previous turns.

- the kick due the leading bunches $\mathrm{j}$ from previous turns.

- the kick due to the trailing bunches $\mathrm{j}$ from previous turns. 
The first contribution is expressed by

$$
\left\langle p_{x k}\right\rangle^{\prime}(n) \sim \frac{\left\langle x_{k}\right\rangle(i)}{\sqrt{(n-i) C}}, \quad i<n .
$$

The second contribution is expressed by

$$
\left\langle p_{x k}\right\rangle^{\prime}(n) \sim \frac{\left\langle x_{j}\right\rangle(i)}{\sqrt{(n-i) C+\Delta s_{k \rightarrow j}}}, \quad i \leq n .
$$

The third contribution is expressed by

$$
\left\langle p_{x k}\right\rangle^{\prime}(n) \sim \frac{\left\langle x_{j}\right\rangle(i)}{\sqrt{(n-i+1) C-\Delta s_{j \rightarrow k}}}, \quad i \leq n .
$$

\section{PRODUCTION AND ANALYSIS}

\subsection{The Task}

We studied the dynamics of the beam at injection and obtained the growth rates of the different bunches in both transverse planes. The study was done as guidance for the design of a transverse feedback system.

First we checked if tracking based on a 7:th order map was representing the correct transverse dynamics, in particular by comparing with a symplectic integrator, i.e. Tracy-2. Fig. 5 is shown as a reference and represents the transverse phase space of a particle tracked with the symplectic integrator. We also tracked the particle using a 7:th order map for different values of the betatron amplitudes. Fig. 6 shows the results for a particle with $5 \mathrm{~mm}$ horizontal amplitude and $1 \mathrm{~mm}$ in the vertical plane. The agreement with the result from the symplectic integrator is good. Fig. 7 is obtained for a $10 \mathrm{~mm}$ oscillation amplitude in the horizontal plane and $1 \mathrm{~mm}$ in the vertical plane. The horizontal phase space is expanding whereas the vertical is contracting. However, the symplectic integrator gives stable motion in both planes, proving that the truncated power series map fails to approximate the dynamics in this case.

The non-linear motion will therefore be studied by using a 7:th order map and a particle with a maximum of $5 \mathrm{~mm}$ oscillation amplitude in the 


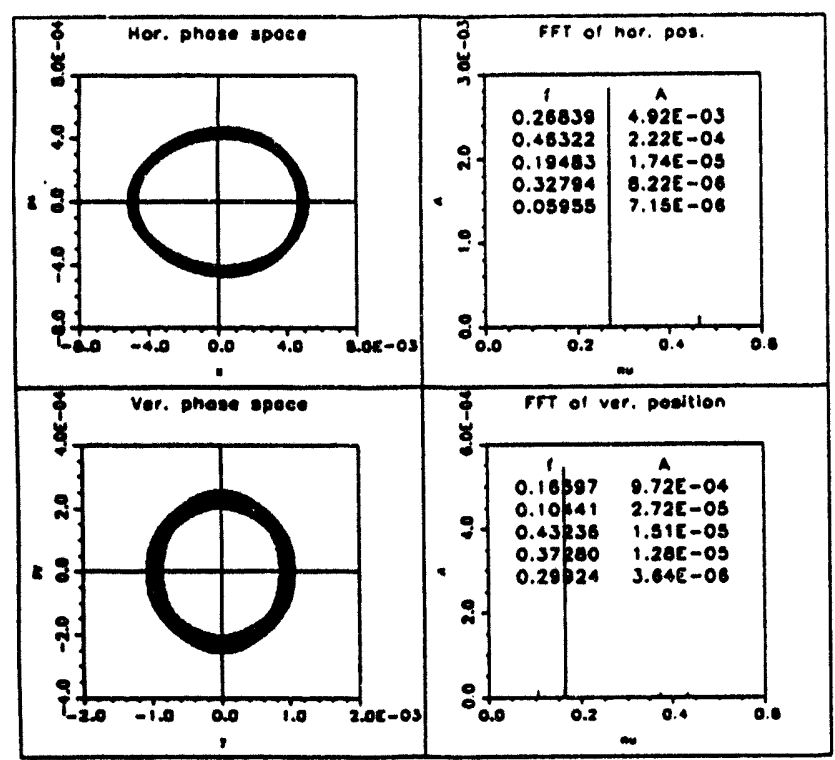

Figure 5: Symplectic integrator: $5 \mathrm{~mm}$ horizontal and $1 \mathrm{~mm}$ vertical amplitude.

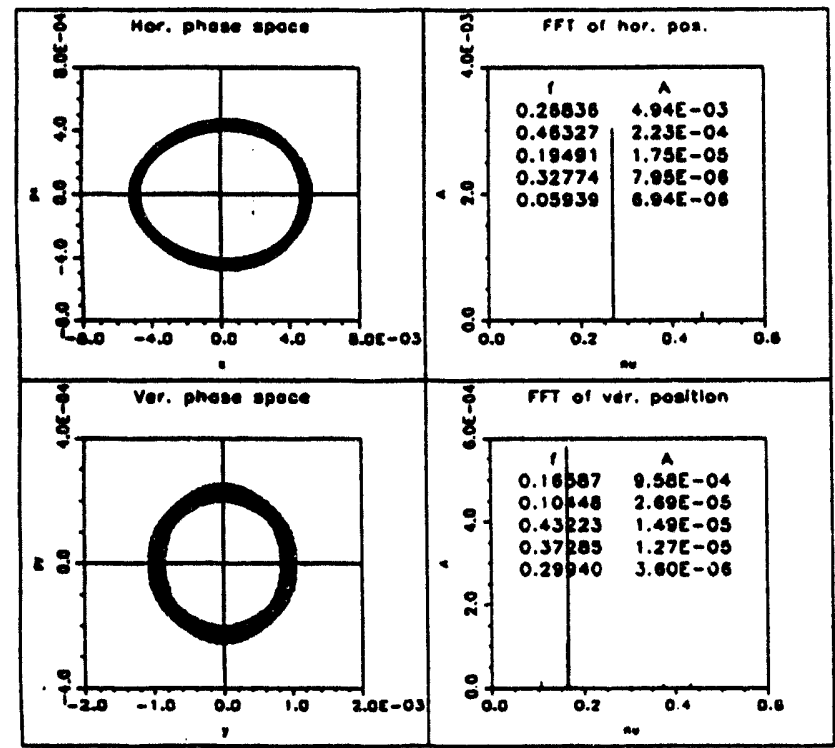

Figure 6: 7:th order map: $5 \mathrm{~mm}$ horizontal and $1 \mathrm{~mm}$ vertical amplitude. 


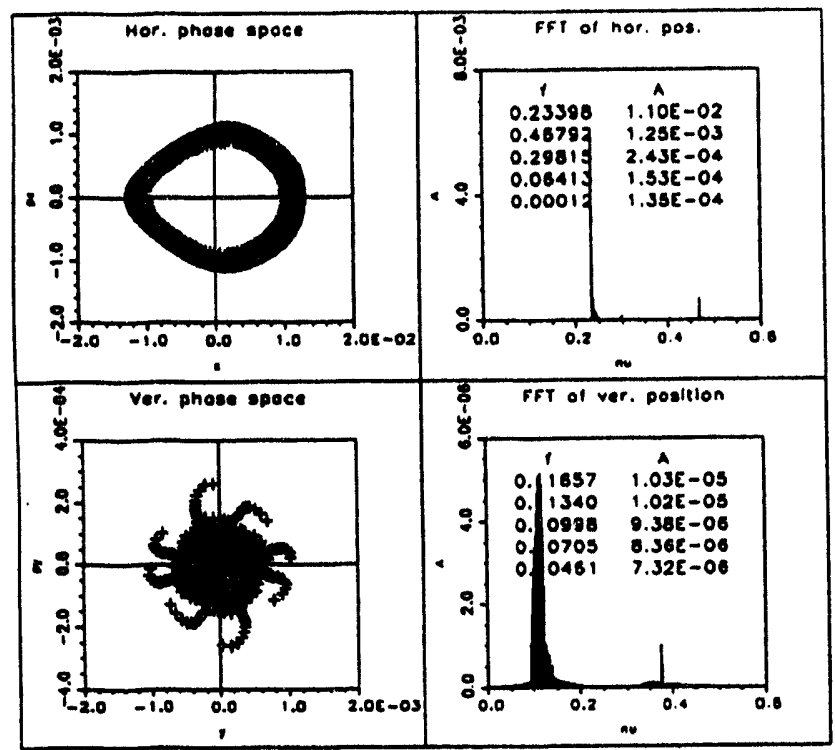

Figure 7: 7:th order map: $10 \mathrm{~mm}$ horizontal and $1 \mathrm{~mm}$ vertical amplitude.

horizontal plane. We tried to reduce the map to $5:$ th order but the dynamics were not consistant with the symplectic integrator. In our case, a $7:$ th order map is therefore needed to study the transverse dynamics of the beam.

\subsection{Linear Map}

Using a linear map, we modeled 4 injected bunches with 6 bunches already stored, assumed to have damped down to zero betatron amplitudes. The bunches are equally spaced around the machine circumference. In both planes, the resistive wall is switched on as well as the HOMs of the cavities. The parameters for the studies are summarized in the following output file: 
Particle energy: $1.5 \mathrm{GeV}$

Tune: $\nu_{x}=14.277, \nu_{y}=8.179$

Beta functions at the kick point: $\beta_{x}=11.296 \mathrm{~m}, \beta_{y}=4.231 \mathrm{~m}$

Total number of bunches: 10

Particles per bunch: $5 \times 10^{9}$

Ring circumference: $196.8 \mathrm{~m}$

Harmonic number: 10

Number of resistive wall kicks: 1

Revolution frequency: $1.52334 \mathrm{MHz}$

Revolution period: $0.656454 \mu \mathrm{s}$

$\mathrm{RF}$ frequency: $499.654 \mathrm{MHz}$

Bucket spacing: $0.60 \mathrm{~m}$

$\mathrm{FF}$ wavelengths between bunches in a train: 1

Bunch spacing within a train: $0.60 \mathrm{~m}$

Initial offset the first displaced bunch " 1 " is $x: 5.0 \mathrm{~mm}, \mathrm{y}: 1.0 \mathrm{~mm}$

First bunch to be displaced: 1

Last bunch to be displaced: 4

Total number of turns: 4096

In order to study the particle growth rate, we examined the following cases

- Result on the tune which is resonant with the first horizontal HOM.

- Result on the tune which is resonant with the second horizontal HOM.

- Result on the tune which is resonant with the third horizontal HOM.

- Result on the tune which is resonant with the fourth horizontal HOM.

Tab. 6 presents the result for bunches experiencing the maximum amplitude growth and Fig. 8 shows the amplitude growth for bunches 1, 5 and 10 in the second case. The amplitude growth of the stored bunches, excited by the injected bunches, is linear with time. The injected bunches are slowly growing exponentially.

We now present the results of a study of the resistive wall effect and compare it with the effect of the cavity HOMs on the beam dynamics. As before, 10 bunches were used, i.e. 4 injected bunches displaced by $x=5$ $\mathrm{mm}$ and $y=1 \mathrm{~mm}$ and 6 stored bunches assumed to be damped down to zero betatron amplitudes. A linear map was used and the macro particles 

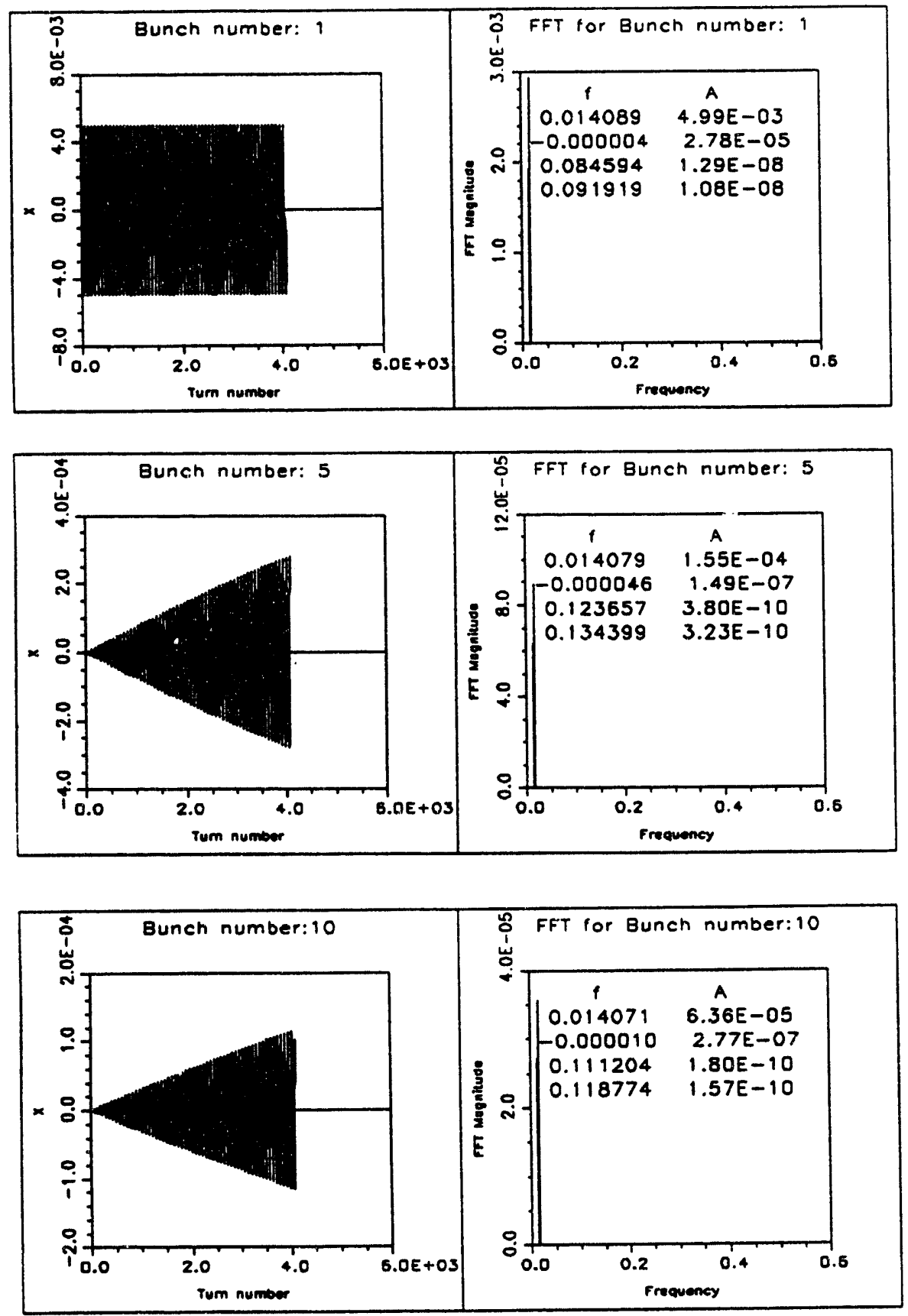

Figure 8: Linear map: Amplitude growth of bunch 1, 5 and 10. 


\begin{tabular}{|c|c|c|c|c|c|c|}
\hline hor. tune & ver. tune & $\begin{array}{c}\text { HOM } \\
{[\mathrm{MHz}]}\end{array}$ & $\begin{array}{c}x_{1-4} \\
{[\mathrm{~mm}]}\end{array}$ & $\begin{array}{c}y_{1-4} \\
{[\mathrm{~mm}]}\end{array}$ & $\begin{array}{c}x_{5-10} \\
{[\mathrm{~mm}]}\end{array}$ & $\begin{array}{c}y_{5-10} \\
{[\mathrm{~mm}]}\end{array}$ \\
\hline 14.21971 & 8.17900 & 810.08 & 12.6837 & 1.0031 & 8.3734 & 0.2351 \\
\hline 14.98591 & 8.17900 & 1122.72 & 5.0505 & 1.0032 & 0.3176 & 0.2367 \\
\hline 14.27700 & 8.60954 & 1121.77 & 5.1203 & 1.0185 & 0.4046 & 0.2239 \\
\hline 14.27700 & 8.32582 & 1801.61 & 5.1198 & 1.0064 & 0.4041 & 0.2276 \\
\hline
\end{tabular}

Table 6: Max. amplitude growth due to HOMs.

\begin{tabular}{|c|c|c|c|c|c|c|}
\hline bunch number & \multicolumn{2}{|c|}{$\begin{array}{c}\text { Res. Wall + Cavity } \\
{[\mathrm{mm}]}\end{array}$} & \multicolumn{2}{c|}{$\begin{array}{c}\text { Res. Wall (1 kicks) } \\
{[\mathrm{mm}]}\end{array}$} & \multicolumn{2}{c|}{$\begin{array}{c}\text { Cavity } \\
{[\mathrm{mm}]}\end{array}$} \\
\hline bunch 1-4 & 5.1196 & 1.0031 & 5.0000 & 1.0044 & 5.1378 & 1.0037 \\
\hline bunch 5-10 & 0.4039 & 0.2351 & 0.3422 & 0.2363 & 0.1665 & 0.0268 \\
\hline
\end{tabular}

Table 7: Max. amplitude growth due to resistive wall and HOMs.

were tracked for 4096 turns. The results are shown in Tab. 7 Comparison was also done between a single thin kick for the resisitive wall effect and 500 . Tab. 8 shows the resulting amplitude for 500 thin kicks using the same initial conditions as before.

\subsection{Non-Linear Map}

Tab. 9 shows a comparison between linear and non-linear map for the second horizontal HOM. Fig. 9 represents the growth of the first bunch (the injected one) and the successive bunches. The amplitudes of these bunches are now beating and the FFT shows a double peak around each tune. In order to understand this behavior, a driven anharmonic oscillator was modeled and the result is presented in Fig. 10. This leads to the conclusion that the

\begin{tabular}{|c|c|c|}
\hline bunch number & \multicolumn{2}{|c|}{$\begin{array}{r}\text { Resistive Wall (500 kicks) } \\
\text { [mm] }\end{array}$} \\
\hline bunch 1-4 & 5.0000 & 1.0000 \\
\hline bunch 5-10 & 0.3312 & 0.2308 \\
\hline
\end{tabular}

Table 8: Max. amplitude growth due to resistive wall. 

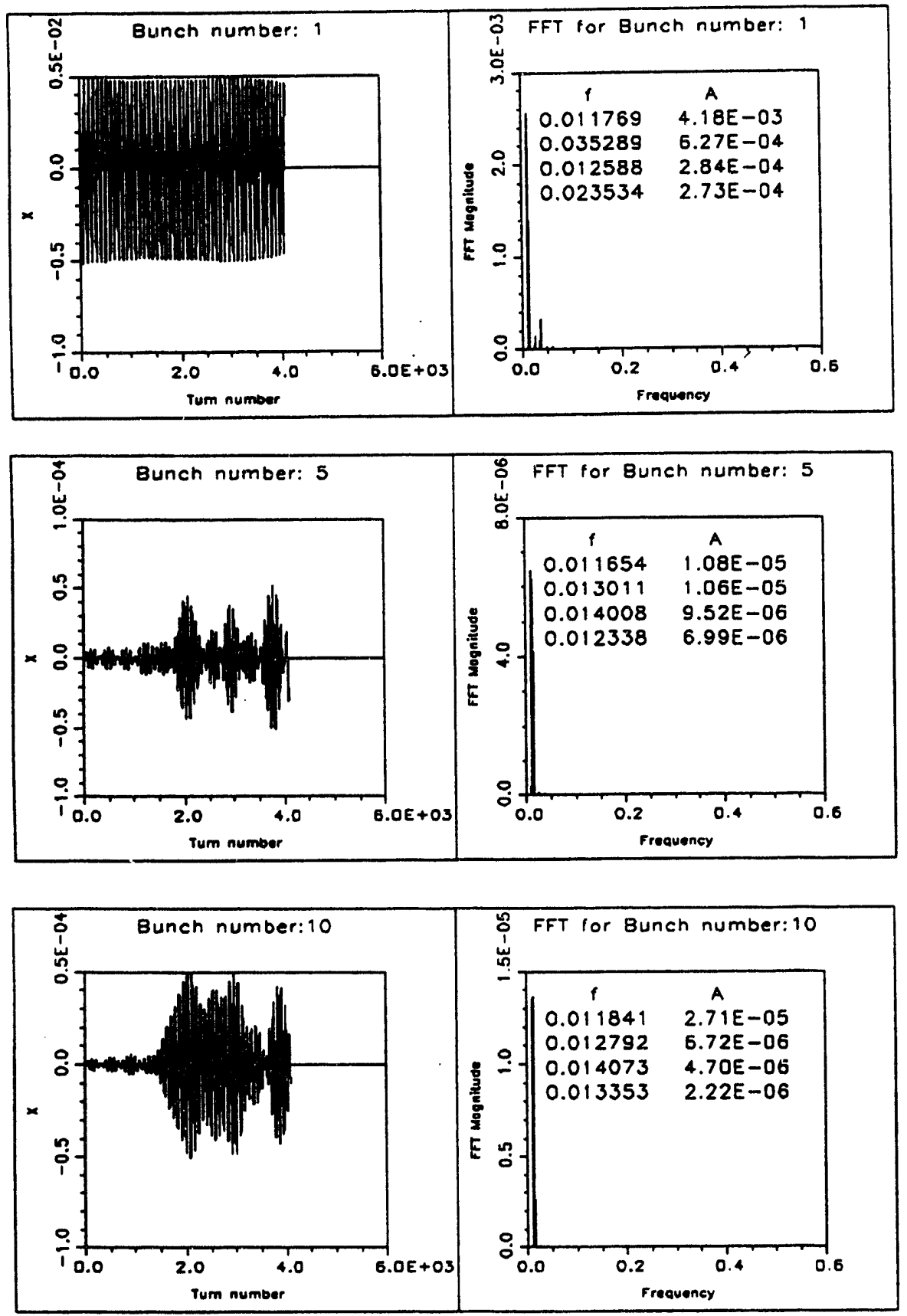

Figure 9: Non-linear map: Amplitude growth of bunch 1, 5 and 10. 


\begin{tabular}{|c|c|c|c|c|c|}
\hline Map & $\begin{array}{c}\text { HOM } \\
{[\mathrm{MHz}]}\end{array}$ & $\begin{array}{c}x_{1-4} \\
{[\mathrm{~mm}]}\end{array}$ & $\begin{array}{c}y_{1-4} \\
{[\mathrm{~mm}]}\end{array}$ & $\begin{array}{c}x_{5-10} \\
{[\mathrm{~mm}]}\end{array}$ & $\begin{array}{c}y_{5-10} \\
{[\mathrm{~mm}]}\end{array}$ \\
\hline Linear & 1122.72 & 5.0505 & 1.0032 & 0.3176 & 0.2367 \\
\hline Non-linear & 1122.72 & 5.3246 & 1.1911 & 0.0546 & 0.0013 \\
\hline
\end{tabular}

Table 9: Max amplitude growth for linear vs non-linear map.

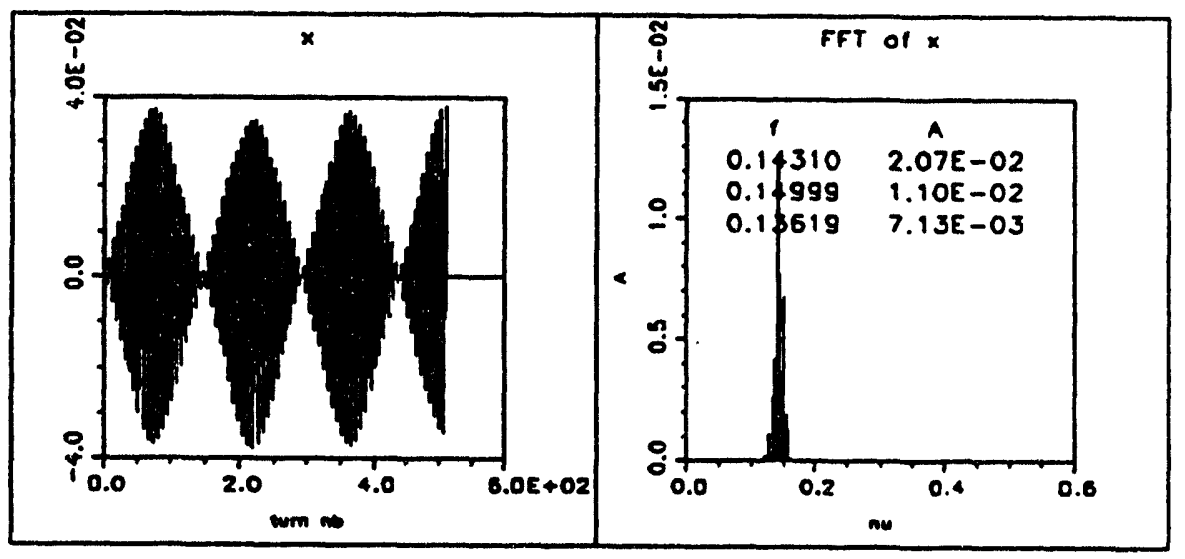

Figure 10: Driven anharmonic oscillator.

beating is a consequence of the amplitude dependent tune shift induced by the sextupoles. In other words, the amplitude dependent tune shift makes the particle slip out of phase relative to the driving field.

In order to compare the simulated linear growth rates with the analytical expression in chapter 1 , we again used 10 bunches, 4 injected with $x=5 \mathrm{~mm}$ and $y=1 \mathrm{~mm}$ and 6 stored assumed to have damped down to zero betatron amplitudes. Only the resistive wall effect was included, using 10 thin kicks. Tab. 10 shows the simulation results for the stored bunches experiencing linear amplitude growth due to the injected beam.

We also studied the growth time for a particular eigen mode of the 4 injected bunches, with only resistive wall effect using 10 wake kicks. For each plane we computed analytically the 4 eigenmodes using LINPACK [24]. One unstable mode was selected and we obtained the corresponding eigenvector 


\begin{tabular}{|c|c|c|c|}
\hline \multicolumn{2}{|c|}{ hor. $[\mathrm{sec}]$} & \multicolumn{2}{c|}{ ver. $[\mathrm{sec}]$} \\
\hline Tracking & Calculated & Tracking & Calculated \\
\hline 0.122 & 0.116 & 0.0902 & 0.0842 \\
\hline 0.0963 & 0.0918 & 0.0727 & 0.0673 \\
\hline 0.0826 & 0.0788 & 0.0631 & 0.0579 \\
\hline 0.0736 & 0.0702 & 0.0516 & 0.0516 \\
\hline 0.0668 & 0.0638 & 0.0470 & 0.0470 \\
\hline 0.0616 & 0.0589 & 0.0479 & 0.0435 \\
\hline
\end{tabular}

Table 10: Linear amplitude growth.

\begin{tabular}{|c|c|c|c|}
\hline \multicolumn{2}{|c|}{ hor. [sec] } & \multicolumn{2}{c|}{ ver. [sec] } \\
\hline Tracking & Calculated & Tracking & Calculated \\
\hline 0.532 & 0.215 & 0.0916 & 0.0605 \\
\hline 0.193 & 0.215 & 0.07 .38 & 0.0605 \\
\hline 0.199 & 0.215 & 0.0567 & 0.0605 \\
\hline 0.233 & 0.215 & 0.0638 & 0.0605 \\
\hline
\end{tabular}

Table 11: Growth times for a single eigenmode.

defining the initial conditions in $\left(x, p_{x}, y, p_{y}\right)$ for the 4 bunches. Tracking with these initial conditions gaven the growth times for the two planes. The results are shown in Tab. 11. The discrepancy between tracking and analytical results is due to numerical roundoff errors, since tracking of a single eigenmode will be particularly sensitive. Note that the resistive wall kick is roughly 6 magnitudes smaller than the average kick from the betatron motion. The expected results were obtained by a scaling test and agreement was found when the impedance was scaled by a factor of $10^{2}-10^{3}$.

\section{MORE ON THE INJECTION PROCESS}

The beam is injected from the booster to the storage ring with very large transverse oscillation amplitudes: $13 \mathrm{~mm}$ in the horizontal plane and $1 \mathrm{~mm}$ in the vertical plane. We studied the behavior of the beam just after the injection without taking the resistive wall effect into account. Fig. 11 shows the results. The tracking was done using a symplectic integrator perturbed 


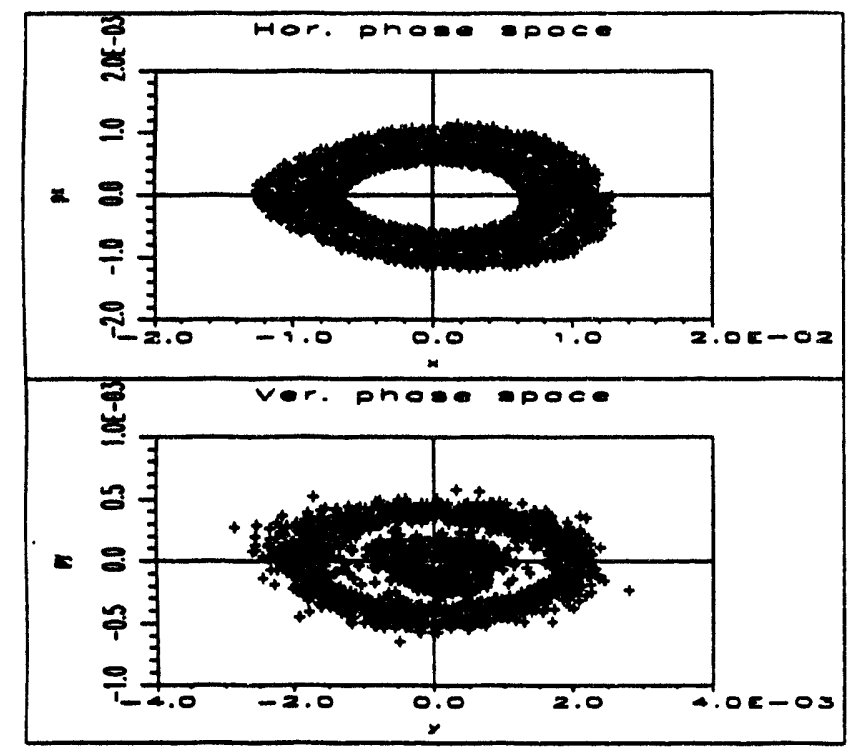

Figure 11: Phase space just after injection.

by synchrotron radiation (damping time $10 \mathrm{msec}$ ) by tracking one damping time, i.e. 15000 turns (with Tracy-2). Initially we observe damping in both planes due to synchrotron radiation and a fair amount of beating due to linear coupling. However, after a few thousand turns there is a sudden increase of amplitude, especially in the vertical plane, after which the particle continues to damp. This is explained by the rather large amplitude dependent tune shift for ALS, originating from a sextupole scheme based on only two families. A particle will therefore cross resonances during its path towards zero amplitude. When this happen, the amplitude will grow until amplitude dependent tune shift moves it away from the resonance. If the resonance is strong enough, the particle may become unstable and hit the beampipe. Otherwise, it continues to damp towards zero but now following a different path in the tune diagram. If no strong resonances are crossed it eventually makes it down to zero. It is clear that the dynamics of the damped motion is very complex and quite different from the naive model that injected particles are smoothly damped to small amplitudes in a few damping times. 


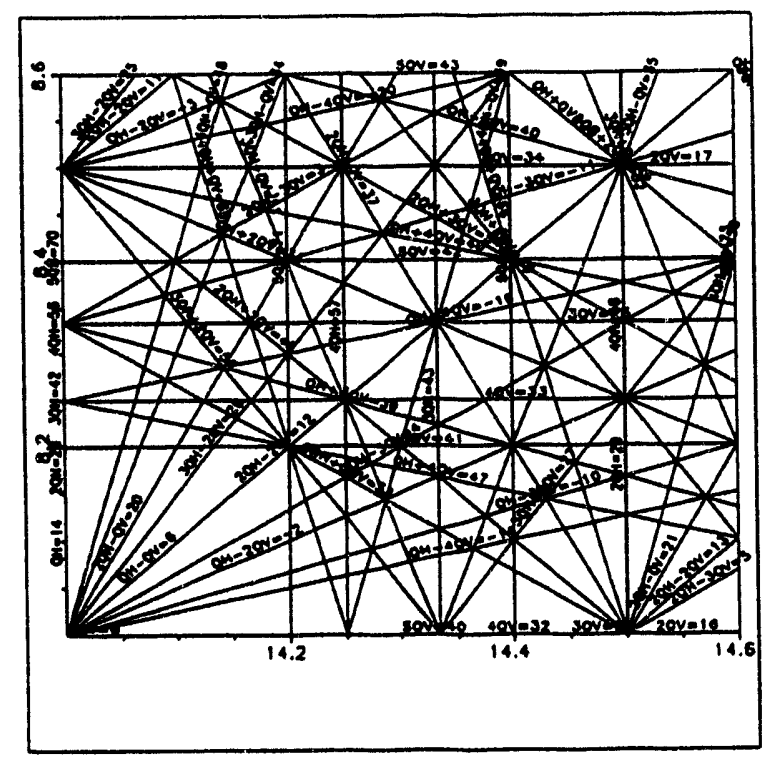

Figure 12: Tune diagram.

\section{OTHER APPLICATIONS}

The code is structured around independent subroutines. It can easily be used for other accelerators by simply changing the input files with the appropriate ring characteristics. Similarly, relevant parameters for resistive wall effect and cavity HOMs are defined in corresponding input files.

In parallel, work has been pursued to develop a computer code [25] for the full 6-dimensional dynamics based on power series maps for the lattice, including models for longitudinal as well as transverse feedback systems. This code has its origin in an early effort to simulate the longitudinal feedback system currently being constructed for the B-factory to be tested at ALS. For easy implementation and support of a powerful user interface, this code has applied modern programming techniques like object oriented programming, in particular Objective-C has been applied. However, the presented Fortran implementation allowed us to reuse and significantly extend the initial Fortran code developed at SLAC. This led to fast protutyping and consistency checks of the dynamical model before migration of the relevant dynamics to the final code. 
An interesting possibility [26] is to add the current model to a general tool like Matrix-X [27] used to study feedback systems in general. The possibility to call $\mathrm{C}$ routines allows for a straightforward integration of the presented model to this environment. This would allow for easy modeling of the whole system, including possible feedback systems, to study limitations due to e.g. quantization noise, saturation effects as well as suitable gain coefficients and overall system performance. Independently, straighforward analytical work [28] has been done by applying classical control theory to study the dynamics of the transverse feedback system currently being tested at ALS. This work is currently being extended to study saturation effects using Matlab $[29,30]$.

\section{SUMMARY AND CONCLUSIONS}

We have derived the linearized transverse equations of motion for the rigid bunch motion in the case of wake fields. A map approach was persued by averaging the equations of motion, in particular by lumping distributed wake fields into a thin kick. This was straightforward using a Hamiltonian formalism. The final equations of motion allows us to consider one turn maps, in particular their power series representation on a computer, to represent the lattice. This allowed for a straightforward generalization to include the non-linear dynamics, synchrotron radiation as well as generalizing to the full 6-dimensional case. In addition, we have also implemented a 2-dimensional wake field model for the HOM in a cavity. The computer implementation was successfully checked against analytical calculations. The tracking code was used to study the non-linear transverse dynamics for ALS as a guide in the design of a related transverse feedback system. These studies showed qualitative changes. in the dynamics of the injection process as well as the stored beam due to non-linear effects from the lattice.

\section{Acknowledgements}

We would like to thank S. Chattopadhyay for consistent support of this work. We would also like to thank $E$. Forest for reading and commenting on the first part and A. Zholents for reading the entire paper and related constructive criticism. 


\section{References}

[1] E. D. Courant and A. M. Sessler Transverse Coherent Resistive Instabilities of Azimuthally Bunched Beams in Particle Accelerators Rev. Sci. Instr. p. 3875 vol. 37, No 10 (1965)

[2] S. Chattopadhyay Some Fundamental Aspects of Fluctuations and Coherence in Charged-Particle Beams in Storage Rings CERN 84-11 (1984).

[3] A. Chao Coherent Instabilities of a Relativistic Bunched Beam AIP Conf. Proc. No 105, (Am. Inst. of Physics, New York, 1982), p. 353.

[4] B. Zotter Electromagnetic Fields: Impedances and Wakes 1987 American Institute of Physics, p. 663.

[5] K. Bane, T. Weiland and P. Wilson Wake Fields and Wake Field Acceleration AIP Conf. Proc. No 127 p. 875, (Am. Inst. of Physics, New York, 1983), Chapter 2.

[6] E. Forest, L. Michelotti, A. Dragt and J. S. Berg Stability of Particle Motion in Storage Rings AIP conf. proc. No 292 (1994).

[7] J. Bengtsson Non-linear Transverse Dynamics for Storage Rings with Aapplications to the Low-Energy Antiproton Ring (LEAR) at CERN CERN 88-05.

[8] A. Chao Physics of Collective Beam Instabilities in High Energy Accelerators (John Wiley \& Sons, Inc. 1993).

[9] B. Zotter and F. Sacherer Transverse Iinstabilities of Relativistic Particle Beams in accelerators and storage rings CERN 77-13, p. 175.

[10] J. Gareyte Beam Observation and the Nature of Instabilities CERN SPS/87-18 (AMS)

[11] K. Bane Wakefield Effects in a Linear Collider, AIP Conf. Proc. No 153, (Am. Inst. of Physics, New York, 1987).

[12] P. Wilson High Energy Electron Linacs; Application to Storage Ring RF Systems and Linear Colliders AIP Conf. Proc. No 87, (Am. Inst. of Physics, New York, 1982), p. 450. 
[13] W. K. H. Panofsky and W. A. Wenzel Some Considerations Concerning the Transverse Deflection of Charged Particles in Radio-Frequency Fields Rev. Sci. Instr. vol. 27, p. 967 (1956).

[14] K. A. Thompson Extension of Coupled-Bunch Ring Simulation to Transverse Case Asymmetric B-factory Collider note ABC-57.

[15] S. Chattopadhyay, priv. comm.

[16] P. M. Morse and H. Feshbach Methods of Theoretical Physics (McGra NHill 1953), part 1, p. 466.

[17] J. Gareyte Observation and Correction of Instabilities in Circular Accelerator CERN SL/91-09 (AP).

[18] 1.2 GeV Synchrotron Radiation Source Conceptual Design Report, July 1986, PUB-5172 Rev.

[19] K. A. Thompson and R. D. Ruth Transverse Coupled-Bunch Instabilities in Damping Rings of High-Energy Linear Colliders SLAC-PUB 4962.

[20] J. Bengtsson TRACY-2 User's Manual unpubl.

[21] E. Forest, unpubl.

[22] J. Corlett, priv. comm. and ALS RF Cavity Measurements to be publ. Nov. 1992.

[23] J. Corlett, private comm.

[24] J. J. Dongarra, C. B. Moler, J. R. Bunch and G. W. Stewart LINPACK User's Guide (Siam, Philadelphia, 1979).

[25] D. Briggs et al. to be publ.

[26] F. Rouse, priv. comm.

[27] Matrix-X Integrated Systems, Inc.

[28] J. Bengtsson, G. Portmann and D. Briggs A Linear Control Theory Analysis of Transverse Coherent Bunch Instabilities Feedback Systems (The Control Theory Approach to Hill's Equation) CBP Tech Note-026, PeP-II AP Note 28-93. 
[29] Matlab Reference Guide The MathWorks, Inc.

[30] J. Bengtsson, G. Portmann and D. Briggs to be publ. 

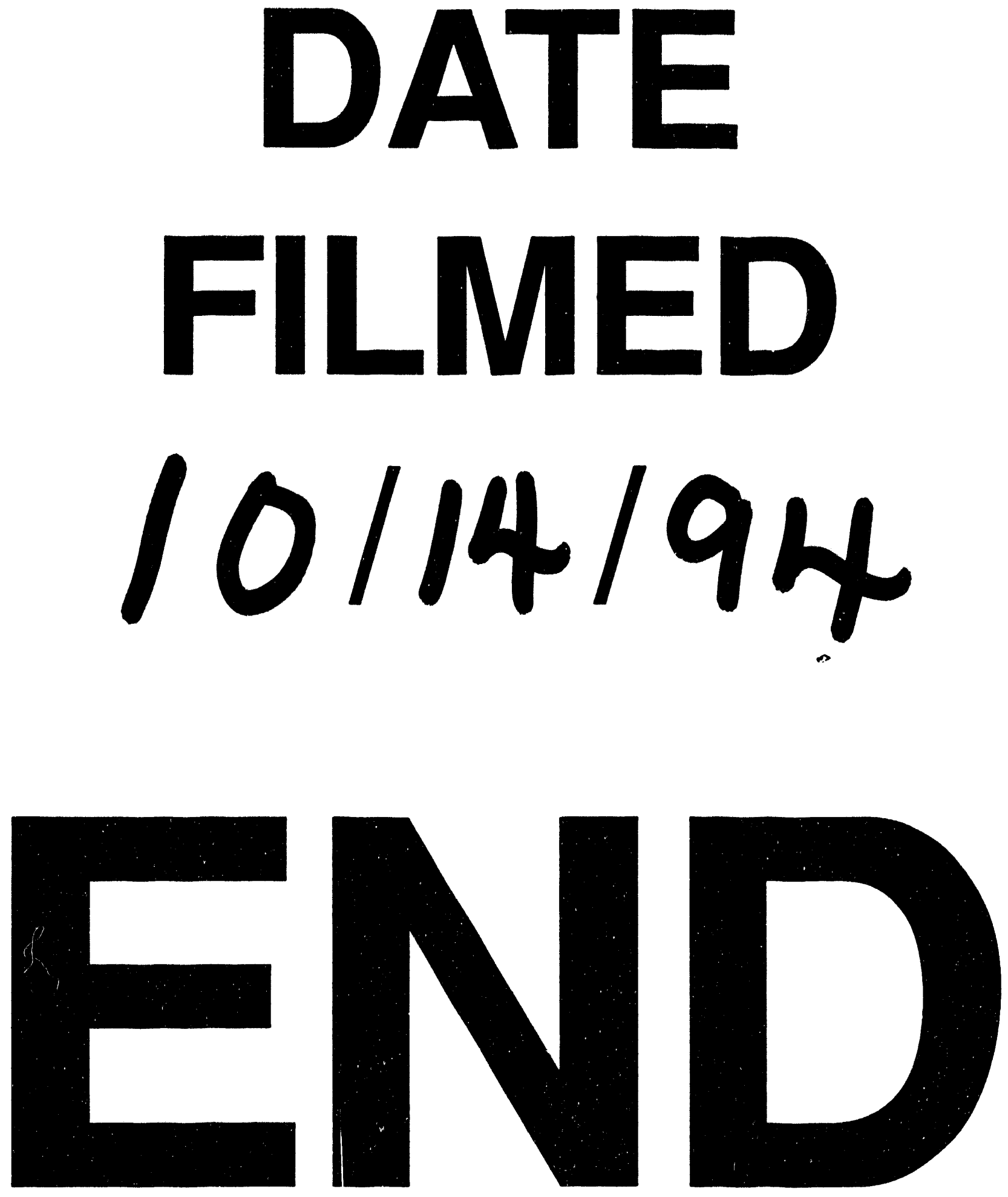


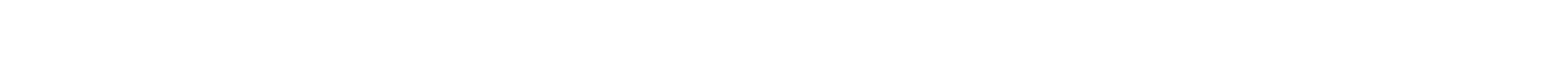

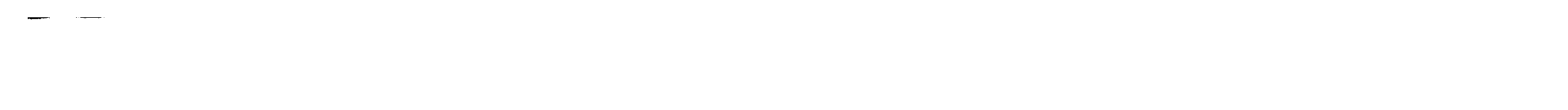$$
-
$$ 